\title{
Actin-Binding Protein Cortactin Promotes Pathogenesis of Experimental Autoimmune Encephalomyelitis by Supporting Leukocyte Infiltration into the Central Nervous System
}

\author{
Maryna Samus, ${ }^{1}$ Yu-Tung Li, ${ }^{1} \oplus^{-}$Lydia Sorokin, ${ }^{2,3}$ Klemens Rottner, ${ }^{4,5}$ and $\odot$ Dietmar Vestweber ${ }^{1,3}$ \\ ${ }^{1}$ Max-Planck Institute for Molecular Biomedicine, D48149 Münster, Germany, ${ }^{2}$ Institute of Physiological Chemistry and Pathobiochemistry D48149 \\ Münster, Germany, ${ }^{3}$ Cells-in-Motion Cluster of Excellence, University of Münster, 48149 Münster, Germany, ${ }^{4}$ Helmholtz Centre for Infection Research, \\ 38124 Braunschweig, Germany, and ${ }^{5}$ Technische Universität Braunschweig, 38106 Braunschweig, Germany
}

Leukocyte entry into the central nervous system (CNS) is essential for immune surveillance but is also the basis for the development of pathologic inflammatory conditions within the CNS, such as multiple sclerosis and its animal model, experimental autoimmune encephalomyelitis (EAE). The actin-binding protein, cortactin, in endothelial cells is an important player in regulating the interaction of immune cells with the vascular endothelium. Cortactin has been shown to control the integrity of the endothelial barrier and to support neutrophil transendothelial migration in vitro and in vivo in the skin. Here we use cortactin gene-inactivated male and female mice to study the role of this protein in EAE. Inducing EAE by immunization with a myelin oligodendrocyte glycoprotein peptide $\left(\mathrm{MOG}_{35-55}\right)$ revealed an ameliorated disease course in cortactin gene-deficient female mice compared with WT mice. However, proliferation capacity and expression of IL-17A and IFN $\gamma$ by cortactin-deficient and WT splenocytes did not differ, suggesting that the lack of cortactin does not affect induction of the immune response. Rather, cortactin deficiency caused decreased vascular permeability and reduced leukocyte infiltration into the brains and spinal cords of EAE mice. Accordingly, cortactin gene-deficient mice had smaller numbers of proinflammatory cuffs, less extensive demyelination, and reduced expression levels of proinflammatory cytokines within the neural tissue compared with WT littermates. Thus, cortactin contributes to the development of neural inflammation by supporting leukocyte transmigration through the blood- brain barrier and, therefore, represents a potential candidate for targeting CNS autoimmunity.

Key words: blood-brain barrier; leukocyte extravasation; multiple sclerosis

Significance Statement

Multiple sclerosis is an autoimmune neuroinflammatory disorder, based on the entry of inflammatory leukocytes into the CNS where these cells cause demyelination and neurodegeneration. Here, we use a mouse model for multiple sclerosis, experimental autoimmune encephalomyelitis, and show that gene inactivation of cortactin, an actin binding protein that modulates actin dynamics and branching, protects against neuroinflammation in experimental autoimmune encephalomyelitis. Leukocyte infiltration into the CNS was inhibited in cortactin-deficient mice, and lack of cortactin in cultured primary brain endothelial cells inhibited leukocyte transmigration. Expression levels of proinflammatory cytokines in the CNS and induction of vascular permeability were reduced. We conclude that cortactin represents a novel potential target for the treatment of multiple sclerosis.

\section{Introduction}

Multiple sclerosis is an autoimmune neuroinflammatory disorder of the CNS, characterized by leukocyte-based demyelination

\footnotetext{
Received May 31, 2019; revised Dec. 4, 2019; accepted Dec. 19, 2019.

Author contributions: M.S. and Y.-T.L. performed research;M.S. and D.V. analyzed data; M.S. wrote the first draft of the paper; Y.-T.L., L.S., and K.R. edited the paper; L.S. and K.R. contributed unpublished reagents/analytic tools; D.V. designed research; D.V. wrote the paper.

This work was supported in part by the Max Planck Society to D.V. and Deutsche Forschungsgemeinschaft SFB/TR128 to D.V.

The authors declare no competing financial interests.

Correspondence should be addressed to Dietmar Vestweber at vestweb@mpi-muenster.mpg.de.
}

and neurodegeneration. The entry of $\mathrm{CD} 4^{+} \mathrm{T}$ cells and monocytes into the CNS are essential steps in the course of this disease and require diapedesis through the specialized endothelium of the blood-brain barrier (BBB). The barrier characteristics of this endothelium protect the CNS from changes in the milieu of the blood and require a higher level of contact integrity between endothelial junctions (Fenstermacher et al., 1988; Sedlakova et al., 1999). In addition, the endothelium of the BBB limits im-

https://doi.org/10.1523/JNEUROSCl.1266-19.2019

Copyright $\odot 2020$ the authors 
A

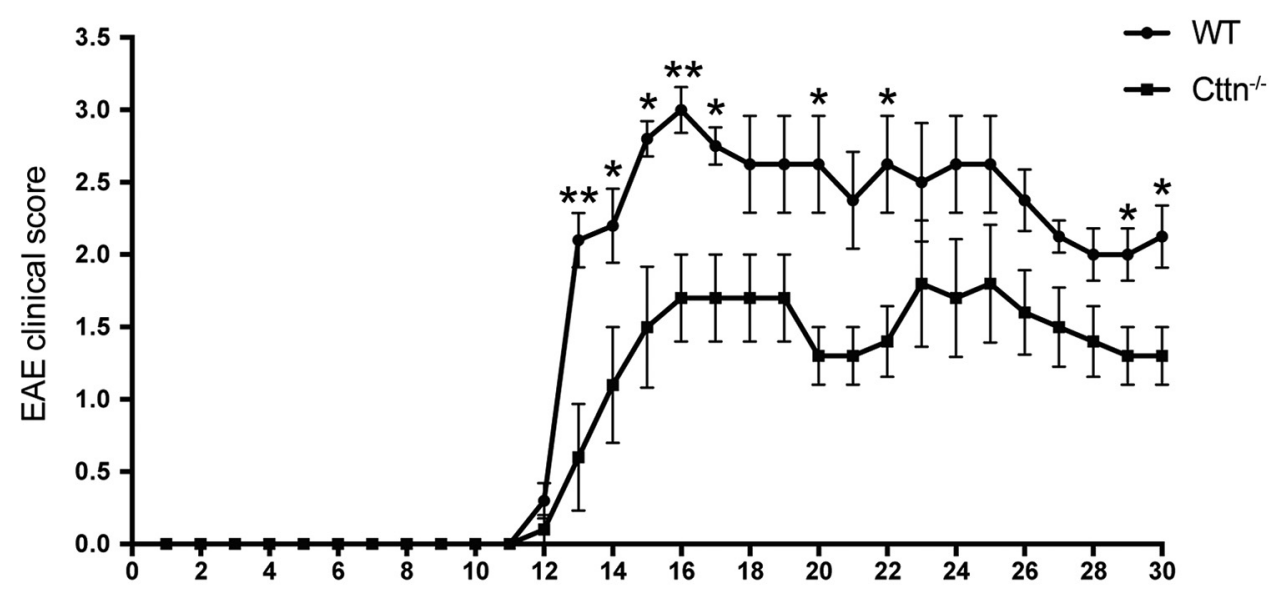

Day after MOG injection

B

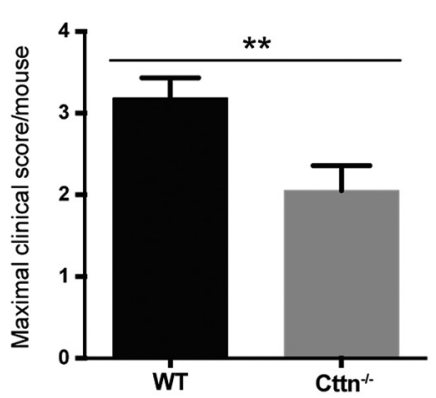

E
C

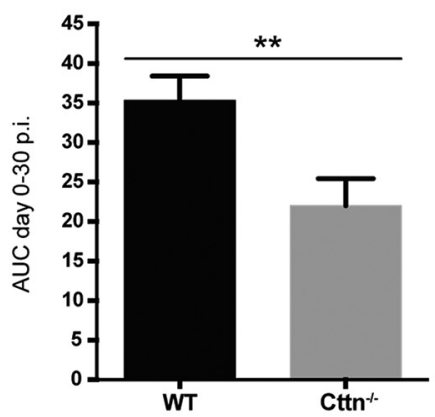

D

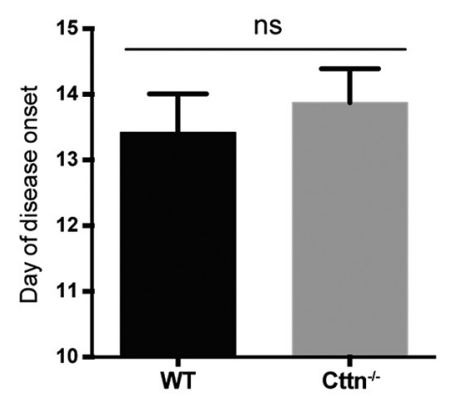

\begin{tabular}{|c|c|c|c|}
\hline & $\begin{array}{c}\text { No clinical score } \\
(\%[\mathrm{n}])\end{array}$ & $\begin{array}{c}\text { Mild clinical score } \\
1-2(\%[\mathrm{n}])\end{array}$ & $\begin{array}{c}\text { Severe clinical } \\
\text { score 3-5 (\% [n]) }\end{array}$ \\
\hline WT & $0(0 / 19)$ & $15,8(3 / 19)$ & $84,2(16 / 19)$ \\
\hline $\mathrm{Cttn}^{--}$ & $15,8(3 / 19)$ & $57,9(11 / 19)$ & $26,3(5 / 19)$ \\
\hline
\end{tabular}

Figure 1. Cortactin deficiency reduces the severity of $\mathrm{MOG}_{35-55^{-}}$induced EAE. $\boldsymbol{A}$, Clinical score of WT mice compared with $\mathrm{Cttn}^{-/-}$mice 0 ver time after $\mathrm{MOG}_{35-55}$ immunization. Data are representative of three independent experiments with $n=5, n=7$, and $n=7$ (total $n=19 /$ group) WT and Cttn ${ }^{-1-}$ mice $\left(^{*} p \leq 0.05\right.$; $\left.{ }^{* *} p \leq 0.01\right)$. B, Maximal clinical score, ${ }^{* *} p=0.0069$. $\boldsymbol{C}$, Area under the curve (AUC) for each group $\left({ }^{* *} p=0.0065\right.$ ) and $(\boldsymbol{D})$ day of disease onset averaged over all 19 mice (not significant (ns): $\left.p=0.5658\right)$. $\boldsymbol{E}$, Numbers and percentages of mice with no, mild, or severe clinical scores in each group. Error bars indicate SEM. Statistics were analyzed with unpaired two-tailed Student's $t$ test.

mune cell entry into the CNS and renders leukocyte diapedesis through this barrier a more demanding task (Sorokin, 2010; Ransohoff and Engelhardt, 2012).

Leukocyte extravasation is mediated by multiple adhesion molecules expressed by endothelial and immune cells and requires the support and dynamic reorganization of the actin cytoskeleton of both cell types (Nourshargh and Alon, 2014; Vestweber, 2015). Current knowledge about the role of endothelial actin-binding proteins in leukocyte adhesion and transendothelial migration is limited (Alon and van Buul, 2017), and only few studies have addressed their contribution to leukocyte extravasation at the BBB (Durieu-Trautmann et al., 1994; LiChunHsieh et al., 2015).

In this study, we focus on cortactin, an actin-binding protein, which is involved in various cellular processes involving regula- tion of membrane dynamics and cell motility, such as cell adhesion, migration, tumor invasion, and endocytosis (Cosen-Binker and Kapus, 2006; Schnoor et al., 2018). Cortactin is expressed in most cell types, except leukocytes, which express the related protein Hematopoietic cell-specific lyn substrate 1 (van Rossum et al., 2005). One of the major molecular functions of cortactin is to bind and activate the actin-filament nucleation complex Arp2/3 (Uruno et al., 2001), which facilitates the branching of F-actin (Mullins et al., 1998).

The role of cortactin in the regulation of the endothelial barrier properties, actomyosin contractility, and leukocyte transendothelial migration (diapedesis) was shown in vitro (Dudek et al., 2004; Jacobson et al., 2006; Yang et al., 2006b; García Ponce et al., 2016) and in a cytokine-induced inflammation model in the cremaster muscle (Schnoor et al., 2011). Cortactin-deficient animals 

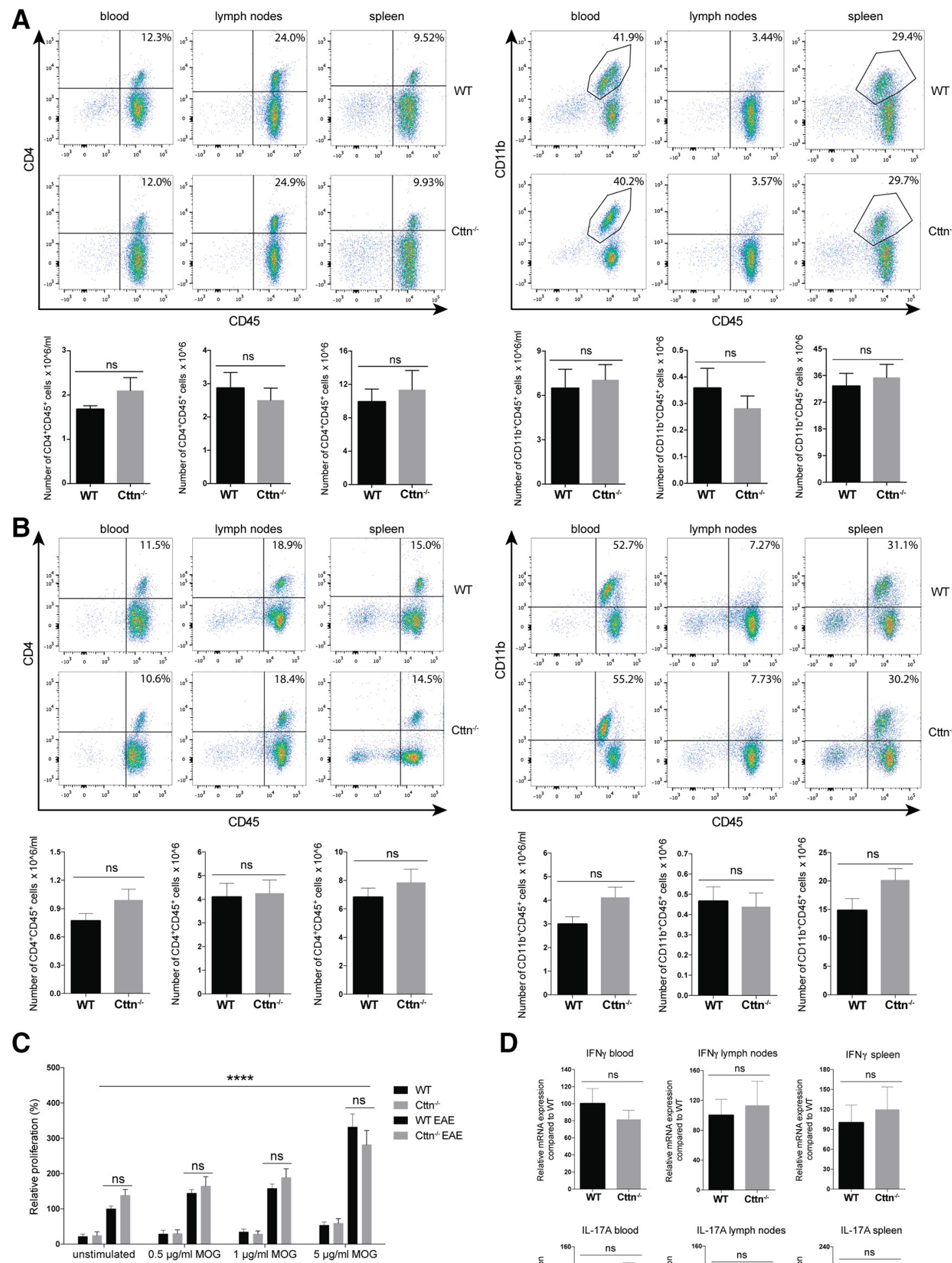

D
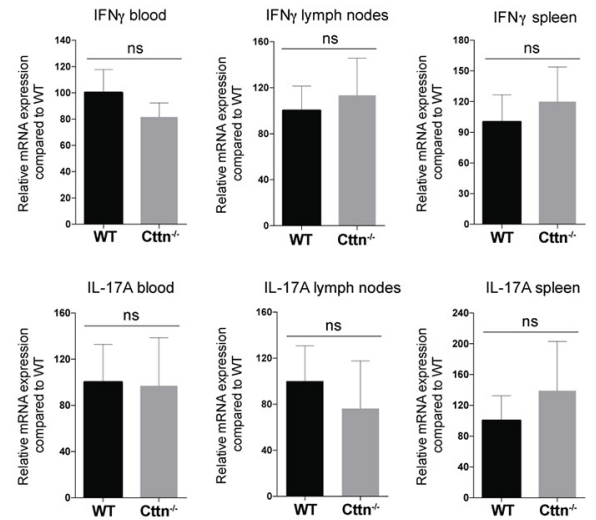

Figure 2. $\quad \mathrm{Cttn}^{-1-}$ and WT mice show comparable peripheral immune responses. WT and $\mathrm{Cttn}^{-1-}$ mice were killed before EAE onset $(\boldsymbol{A})$ or at peak disease (day 16$)(\boldsymbol{B})$, and the number of $\mathrm{CD} 45^{+} \mathrm{CD} 4{ }^{+} \mathrm{T}$ cells and $\mathrm{CD} 45^{+} \mathrm{CD} 11 \mathrm{~b}^{+}$myeloid cells was measured in blood, lymph nodes, and spleen by flow cytometry. Graphs represent results from two independent experiments with $n=$ 4 and $n=6$ (total $n=10 /$ group) WT mice and $\left(\operatorname{ttn}^{-1-}\right.$ mice $(\boldsymbol{A})$; and from three independent experiments with $n=6, n=3$, and $n=5$ (total $n=14 /$ group) WT mice and $C t n^{-1-}$ mice (B). C, Healthy or EAE WT and $\mathrm{Cttn}^{-1-}$ mice were killed on day $12 \mathrm{p.i.}$, splenocytes were isolated and restimulated with different concentrations of $\mathrm{MOG}_{35-55}$ peptide for $72 \mathrm{~h}$; cell proliferation was determined by colorimetric BrdU proliferation assay. Graphs represent mean \pm SEM of three independent experiments with $n=2 /$ experiment $(\operatorname{total} n=6)$ healthy WT and $\mathrm{Cttn}{ }^{-1-}$ mice and $n=$ $3, n=4$, and $n=4$ (total $n=11 /$ group) WT or $n=4 /$ experiment (total $n=12 /$ group) $\mathrm{Cttn}^{-1-}$ EAE-subjected mice. D, Total RNA from CD45 ${ }^{+}$cells in the blood, lymph nodes, and spleens was isolated, and IFN $\gamma$ and IL-17A expression profiles were determined by qRT-PCR; data were normalized to the expression of GAPDH. Expression levels in WT (Figure legend continues.) 
have increased basal vascular permeability in the skin and show enhanced sensitivity to permeability-inducing inflammatory mediators, such as histamine; however, neutrophil transmigration is reduced (Schnoor et al., 2011). In accordance with these data, in vitro cortactin, together with F-actin and ICAM-1, surrounds transmigrating polymorphonuclear leukocytes and facilitates their adhesion and subsequent extravasation through human umbilical vascular endothelial cell monolayers (Yang et al., 2006b).

Here, we have analyzed the potential relevance of cortactin in the endothelial barrier function of the BBB and for the development of the clinical symptoms of experimental autoimmune encephalomyelitis (EAE). We found that cortactin gene inactivation reduced entry of $\mathrm{CD} 4{ }^{+} \mathrm{T}$ cells and myeloid cells into the brain and spinal cord parenchyma; and at the same time, vascular permeability induction in the inflamed CNS was reduced. These protective effects decreased inflammation and demyelination within the CNS and significantly lowered EAE clinical symptoms. This implicates cortactin as an important player in leukocyte recruitment to the CNS and suggests that it may represent a potential target in the treatment of autoimmune neuroinflammatory diseases, such as multiple sclerosis.

\section{Materials and Methods}

Mice. Cortactin-deficient mice (hereafter referred to as $\mathrm{Cttn}^{-1-}$ ) were generated as previously described (Schnoor et al., 2011). For controls, age- and gender-matched littermates or C57BL/6 mice were used. Experiments for Figure 1 were performed only with female mice, whereas other experiments were done with mice of both sexes. All experiments were conducted according to German animal welfare guidelines and were approved by the Landesamt fuer Natur, Umwelt, und Verbraucherschutz Nordrhein-Westfalen.

Antibodies. The following primary antibodies were used: anticortactin (clone 4F11, Millipore), anti-claudin-5-AlexaFluor-488 (clone 4C3C2, Invitrogen), anti-occludin (ab31721, Abcam), anti-VE-cadherin (VE42) (Broermann et al., 2011), anti-ZO-1 (40-2200, Invitrogen), rabbit serum against pan-laminin (Sixt et al., 2001), rat anti-CD45, FITCconjugated rat anti-CD4, anti-CD4-BV421 (\#100544), FITC-conjugated rat anti-CD8, FITC-conjugated rat anti-Ly6G, FITC-conjugated rat antiCD11b, APC-conjugated rat anti-CD45, anti-T-bet (\#644804), antiROR $\gamma \mathrm{t}$ (\#12-6988-82, Invitrogen), anti-IL-17A (\#506910), IFN- $\gamma$ (\#505826), and anti-ICAM1 (YNI-1.1). FITC-conjugated rat anti-IgG2a and -rat anti-IgG2b, APC-conjugated rat anti-IgG2, and rabbit antiFc $\gamma$ RII+III (2.4G2, hybridoma provided by Prof. Alf Hamann). All primary antibodies, if not stated otherwise, were purchased from BD Pharmingen. The following secondary antibodies were used: AlexaFluor488 donkey anti-rabbit, AlexaFluor-488 donkey anti-mouse, and AlexaFluor-647 donkey anti-rat. All secondary antibodies were purchased from Invitrogen.

Induction of active EAE. For induction of active EAE, 8- to 11-week-old mice were used. For controls, age- and gender-matched littermates or C57BL/ 6 mice were used. On day 0 , mice were immunized subcutaneously with $20 \mu \mathrm{g}$ myelin oligodendrocyte glycoprotein peptide $\left(\mathrm{MOG}_{35-55}\right)$ (Sigma Millipore) per mouse emulsified in complete Freund's adjuvant containing Mycobacterium tuberculosis H37RA (Difco). Pertussis toxin (200 ng) (Sigma Millipore) was injected intraperitoneally on day 0 and day 2. Mice were observed daily for up to $30 \mathrm{~d}$ after immunization. The

\section{$\leftarrow$}

(Figure legend continued.) mice were set as 100\%. Graphs represent results from three independent experiments with $n=5 /$ experiment (total $n=15 /$ group) WT mice and $\mathrm{Cttn}^{-1-}$ mice. C, Statistics were analyzed with two-way ANOVA (MOG concentration, $F_{(3,123)}=10.80$, $p<0.001$; EAE status and genotype, $F_{(3,123)}=31.06, p<0.001$; interaction, $F_{(9,123)}=2.266$, $p=0.022$ ) followed by Tukey test (all comparisons of $5 \mu \mathrm{g} / \mathrm{ml}$ MOG vs other concentrations, $\left.{ }^{* * * *} p \leq 0.0001\right)$. Statistics for the rest of the graphs was analyzed with unpaired two-tailed Student's $t$ test. ns $=$ not significant. clinical scores were assigned based on the following criteria: 0 , no signs of disease; 0.5 , loss of the tail tone or abnormal gate; 1 , flaccid tail and absence of curling at the tip when picked up; 1.5 , completely flaccid tail and mild hindlimb weakness; 2 , hindlimbs are severely weak, mouse is able to move in a waddling gait; 2.5 , hindlimbs are partially paralyzed with some movement observed; 3 , hindlimbs and lower back are completely paralyzed; 3.5 , hindlimbs and lower back are completely paralyzed, weakness in the forelimbs; 4 , forelimbs are additionally paralyzed, mouse cannot move; and 5, death. Dead mice were not included in clinical score measurements. Mice were supplied with Diet Gel Recovery (Clear $\mathrm{H}_{2} \mathrm{O}$ ), serving as both a food and liquid source. To reduce suffering, mice reaching Stage 4 were killed.

FACS. Cells from lymph nodes and spleens were isolated by sieving through $70 \mu \mathrm{m}$ cell strainers, and the single-cell suspensions were kept on ice-cold PBS. Peripheral blood was collected by cardiocentesis. Erythrocytes were lysed with ACK lysis buffer (Thermo Fisher Scientific) for 3 min at room temperature. For a collection of immune cells from the brain and the spinal cord, mice were perfused with ice-cold PBS before tissue removal; cells were collected by sieving through $100 \mu \mathrm{m}$ cell strainers, followed by separation into neuronal and leukocyte populations by discontinuous density gradient centrifugation using isotonic Percoll (GE Healthcare). The leukocyte total number was counted (CASY, Schärfe System). For relative quantification of leukocyte subtypes, mouse Fcreceptors were blocked with anti-Fc $\gamma \mathrm{RII}+\mathrm{III}$, and cells were stained with the cell-specific antibodies. To distinguish between $\mathrm{CD} 11 \mathrm{~b}^{+}$infiltrated cells and microglia from the brain, we gated on the CD45 high and $\mathrm{CD} 45^{\text {low }}$ populations, respectively. Flow cytometry was performed with the FACSCanto II (Becton Dickinson) and data evaluated with FlowJo 7.2.5. For flow cytometric analysis of T-cell cytokine expression, splenocytes and leukocytes from brain and spinal cord were isolated from mice at the peak of EAE. Cells were stimulated for $4 \mathrm{~h}$ at $37^{\circ} \mathrm{C}$ with $500 \mathrm{ng} / \mathrm{ml}$ phorbol 12-myristate 13 -acetate and $1 \mu \mathrm{g} / \mathrm{ml}$ ionomycin in the presence of $5 \mu \mathrm{g} / \mathrm{ml}$ protein transport blocker brefeldin A. After stimulation, cells were stained with anti-CD4-BV421 followed by fixation and permeabilization (Foxp3/Transcription Factor Staining Buffer Set, eBioscience) according to the manufacturer's instructions. Transcription factors T-bet and ROR $\gamma \mathrm{t}$, and intracellular cytokines IL-17A and IFN- $\gamma$ were stained. Cells were acquired using FACS CytoFlex S (Beckman Coulter), and data were analyzed with FlowJo 7.2.5.

In vitro cell proliferation assay. For assessment of cell proliferation, WT and $\mathrm{Cttn}^{-1-}$ mice were immunized with $\mathrm{MOG}_{35-55}$ as described above. At day 10 post immunization (p.i.), mice were killed and spleens were removed. Single-cell suspensions were obtained by sieving the spleens through the $40 \mu \mathrm{m}$ strainer. Cells were cultured in 96-well plates at $1 \times$ $10^{5}$ cells/well in RPMI 1640 medium, supplemented with $10 \%$ heatinactivated FBS, $2 \mathrm{~mm}$ L-glutamine, $100 \mathrm{U} / \mathrm{ml}$ penicillin-streptomycin, $1 \%$ nonessential amino acids, $1 \mathrm{~mm}$ sodium pyruvate, and $0.5 \mathrm{~mm}$ $\beta$-mercaptoethanol. Splenocytes were incubated for $72 \mathrm{~h}$ after isolation in the absence or presence of $\mathrm{MOG}_{35-55}(0.5,1$, and $5 \mu \mathrm{g} / \mathrm{ml})$. Cell proliferation assays were performed using a BrdU cell proliferation kit (Merck) according to the manufacturer's instructions. BrdU reagent was added for the final $24 \mathrm{~h}$. Incorporation of BrdU reagent was measured on a Synergy 2 microplate reader (BioTek) at a wavelength of $450 \mathrm{~nm}$. All experiments were performed in triplicate.

Determination of $m R N A$ expression levels using quantitative real-time RT-PCR. Mice were killed at peak EAE and perfused with ice-cold PBS. Total RNA from the brains and the spinal cords was extracted using Trizol (Invitrogen). RT-PCR was performed using SuperScript II Reverse Transcriptase kit (Invitrogen) according to the manufacturer's instructions in a reaction volume of $20 \mu \mathrm{l}$. cDNA samples were subjected to qRT-PCR using iTaq Universal SYBR Green Supermix (Bio-Rad) and amplification was performed using 7300 Real Time PCR System (Applied Biosystems). For qRT-PCR amplification of cDNA, the following primers (Sigma Millipore) were used: CCR2 forward: ATTCTCCACACCCTGTTTCG and CCR2 reverse: CTGCATGGCCTGGTCTAAGT; CXCL1 forward: GTTCCAGCACTCCAGACTCC and CXCL1 reverse: TGGGG ACACCTTTTAGCATC; GAPDH forward: GCCACCCAGAAGACTGTGGAT and GAPDH reverse: GGGATGACCTTGCCCACAG; IFN $\gamma$ forward: CTGGAGGAACTGGCAAAAGG and IFN $\gamma$ reverse: CTGGAC- 
A

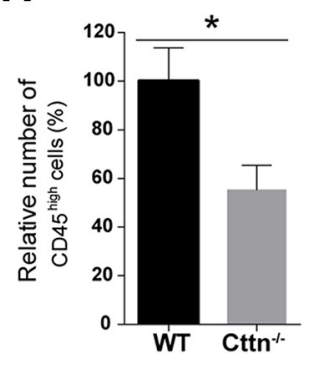

D

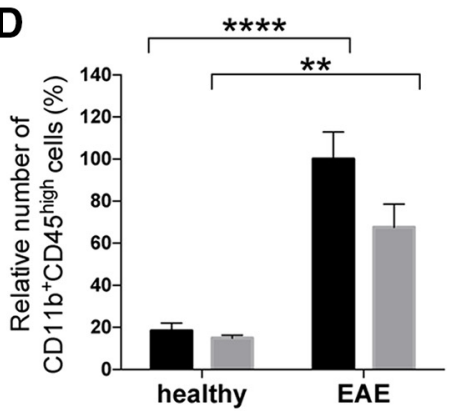

G

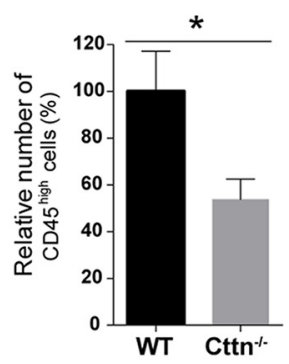

J

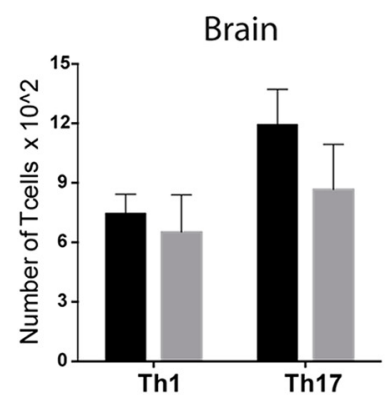

M

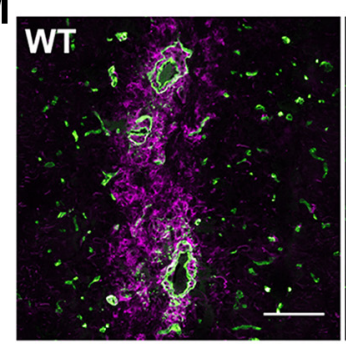

B

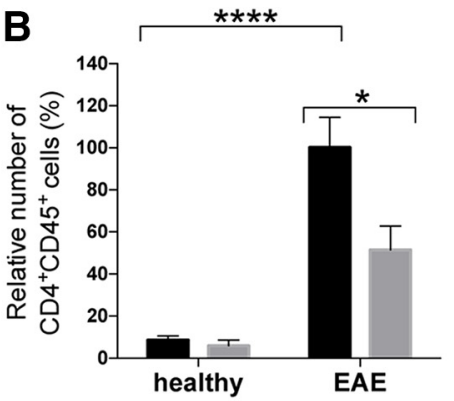

E

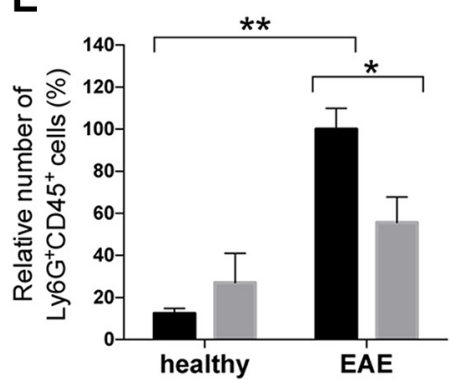

H

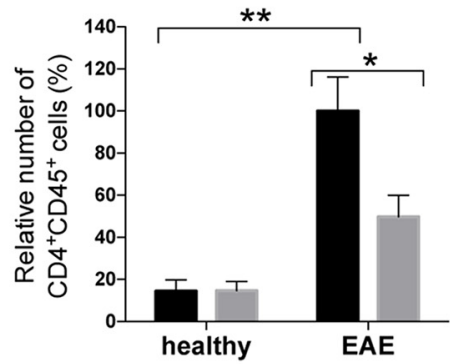

K

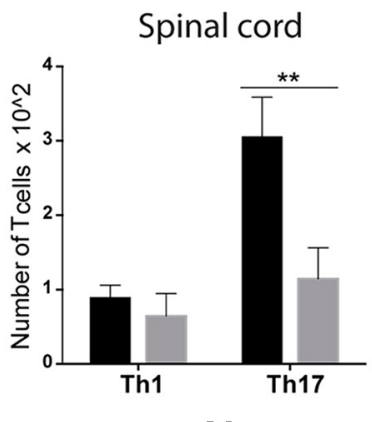

N

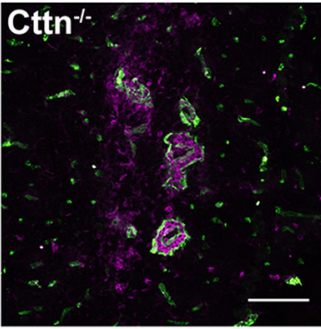

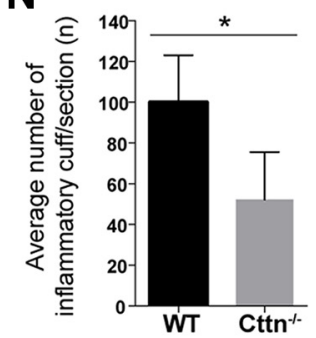

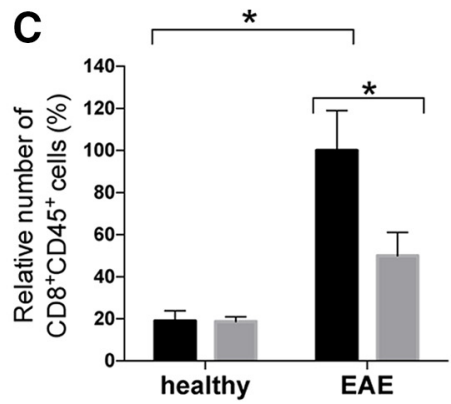

F

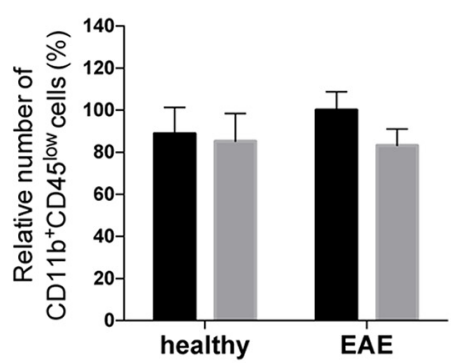

I

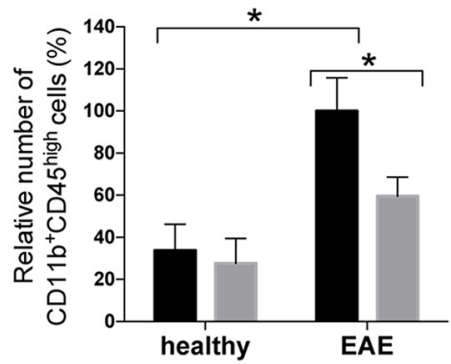

L

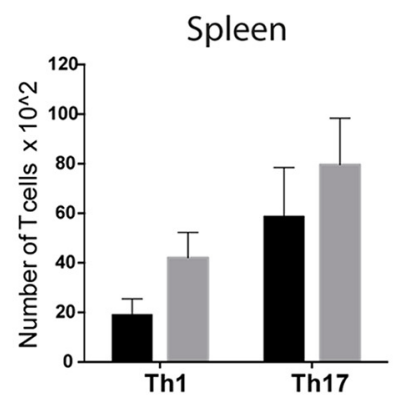

0

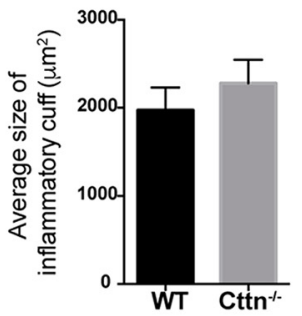

Figure 3. Leukocyte infiltration and numbers of inflammatory cuffs are reduced during EAE in the absence of cortactin. WT or $\mathrm{Cttn}^{-1-}$ mice were killed when WT mice reached peak EAE; brains were isolated and cell numbers were determined. The total number of CD45 high infiltrated leukocytes in WT brains was gated by flow cytometry and set as $100 \%(\boldsymbol{A})$. Subsequently, the proportions

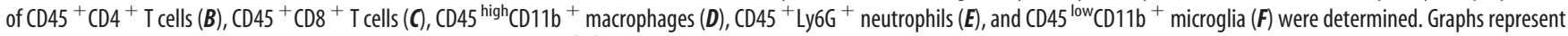
proportions of total $\mathrm{CD} 45^{+}$leukocytes, $\mathrm{CD} 45^{+} \mathrm{CD} 4{ }^{+} \mathrm{T}$ cells, and $\mathrm{CD} 45^{\text {high }} \mathrm{CD} 11 \mathrm{~b}{ }^{+}$macrophages and are mean \pm SEM of three independent experiments with $n=2, n=2$, and $n=4$ (total $n=$ 8/group) healthy WT and (ttn ${ }^{-1-}$ mice; $n=3, n=4$, and $n=5$ (total $n=12$ ) EAE WT mice; and $n=3, n=4$, and $n=4$ (total $n=11$ ) EAE Cttn ${ }^{-1-}$ mice. Graphs shown for CD45 ${ }^{+}$CD8 ${ }^{+}$ $\mathrm{T}$ cells and $\mathrm{CD}_{4} 5^{+} \mathrm{LyGG}^{+}$neutrophils are mean \pm SEM of two independent experiments with $n=3$ healthy WT and $\mathrm{tttn}^{-1-}$ mice; $n=4$ /experiment (Figure legend continues.) 
CTGTGGGTTGTTGA; IL- $1 \beta$ forward: ATCCCAAGCAATACCCA AAG and IL- $1 \beta$ reverse: GTGCTGATGTACCAGTTGGG; IL-6 forward: CCTTCCTACCCCAATTTCCAAT and IL-6 reverse: AACGCACTAGGTTTGCCGAGTA; IL-17A forward: CGCAAAAGTGAGCTCCAGA and IL-17A reverse: TGAGCTTCCCAGATCACAGA; MCP-1 forward: GCATCTGCCCTAAGGTCTTC and MCP-1 reverse AAGTGCTTGAGGTGGTTGTG; RANTES forward: TCTTGCAGTCGTGTTTGTCA and RANTES reverse: CAGGGAAGCGTATACAGGGT; TNF $\alpha$ forward: AGCCCCCAGTCTGTATCCTTCT and TNF $\alpha$ reverse: AAGCCCATTT GAGTCCTTGATG; VE-cadherin forward: TCCTCTGCATCCTCAC TATCACA and VE-cadherin reverse: GTAAGTGACCAACTGCTCGT GAAT; claudin-5 forward: GTGGAACGCTCAGATTTCAT and claudin-5 reverse: TGGACATTAAGGCAGCATCT; occludin forward: AGACCC AAGAGCAGCCAAAG and occludin reverse: GGAAGCGATGAAGCAGAAGG; ZO-1 forward: CCACCTCTGTCCAGCTCTTC and ZO-1 reverse: CACCGGAGTGATGGTTTTCT. The cycling conditions were as recommended by the manufacturer: enzyme activation at $50^{\circ} \mathrm{C}$ for $2 \mathrm{~min}$, initial denaturation at $95^{\circ} \mathrm{C}$ for $10 \mathrm{~min}$, followed by 40 cycles of denaturation at $95^{\circ} \mathrm{C}$ for $15 \mathrm{~s}$ and annealing/elongation at $60^{\circ} \mathrm{C}$ for $1 \mathrm{~min}$. Gene expression was normalized to GAPDH and analyzed using the $\Delta \Delta \mathrm{Ct}$ method. Results were expressed relative to those obtained with WT samples.

In vitro static transmigration assay. A total of $4 \times 10^{4}$ mouse brain microvascular endothelial cells (MBMECs)/well of $3 \mu \mathrm{m}$ transwells (Corning Life Sciences), coated with $40 \%$ gelatin and $0.1 \mathrm{mg} / \mathrm{ml} \mathrm{fi-}$ bronectin (Sigma Millipore) were grown for $4-5 \mathrm{~d}$ and stimulated $16 \mathrm{~h}$ before the assay with $5 \mathrm{~nm}$ TNF $\alpha$ (Peprotech). OTII-OVA T cells were stimulated for $2 \mathrm{~d}$ with $1.5 \% \mathrm{IL}-2$ and added $3 \times 10^{4}$ cells/transwell on

$\leftarrow$

(Figure legend continued.) (total $n=8 /$ group) EAEWT and EAE $\left(\mathrm{ttn}^{-1-}\right.$ mice. G-I, Same as for $\boldsymbol{A}-\boldsymbol{F}$, except that spinal cords instead of brains were analyzed. $\boldsymbol{G}$, Results are from three independent experiments with $n=4, n=5$, and $n=6$ (total $n=15$ ) EAE WT mice and $n=$ $4, n=4$, and $n=6$ (total $n=14$ ) EAE Cttn ${ }^{-I-}$ mice. $\boldsymbol{H}, \boldsymbol{I}$, Results are from three independent experiments with $n=2$ mice/experiment (total $n=6 /$ group) healthy WT and $\mathrm{Ctnn}^{-1-}$ mice; $n=4, n=5$, and $n=6$ (total $n=15$ ) EAE WT mice and $n=4, n=4$, and $n=6$ (total $n=$ 14) EAE $\left(t t^{-1-}\right.$ mice. J-L, Leukocytes were isolated from (J) brains, $(\boldsymbol{K})$ spinal cords, and $(\boldsymbol{L})$ spleens of WT or $\mathrm{Cttn}^{-1-}$ mice at the peak of EAE and assessed for CD4 ${ }^{+} \mathrm{T}_{\text {-bet }}{ }^{+}$IFN- $\gamma^{+}$Th1 and $\mathrm{CD} 4^{+}{ }^{+} \mathrm{ROR} \gamma \mathrm{t}^{+} \mathrm{IL}-17 \mathrm{~A}{ }^{+}$Th17 cells populations. Cell numbers were counted by FACS. Results shown for brain and spinal cord are from two independent experiments with $n=4, n=$ 5 (total $n=9 /$ group) WT and $\mathrm{Ctn}^{-1-}$ mice; results shown for spleen are from 2 independent experiments with $n=4, n=5$ (total $n=9$ ) WT and $n=4$ each (total $n=8) \mathrm{Cttn}^{-1-}$ mice. $M$, Cryosections of brains isolated from mice treated as above (isolated at peak stage of EAE in WT mice) were stained with anti-CD45 for leukocytes (magenta) and with pan-laminin antibodies (green) for identification of perivascular cuffs. Scale bar, $100 \mu \mathrm{m}$. $\boldsymbol{N}$, The number of perivascular cuffs in four distinct sections per mouse was counted in three independent experiments with $n=5, n=5$, and $n=6$ WT mice and $\mathrm{Cttn}^{-1-}$ mice (total $n=16 /$ group). $\boldsymbol{0}$, Average size of inflammatory cuffs was measured in 10 WT (71 cuffs in total) and 5 (ttn ${ }^{-1-}$ mice (41 cuffs in total). Statistics: Unpaired two-tailed Student's $t$ test was used for $(A)$ $\left.{ }^{*} p=0.0174,(\boldsymbol{G}){ }^{*} p=0.0273,(\boldsymbol{K}){ }^{* *} p=0.012,(\boldsymbol{O}) p=0.44\right) . \boldsymbol{N}$, Mann-Whitney $U$ test, ${ }^{*} p=0.0158$. B. Two-way ANOVA (healthy/EAE, $F_{(1,34)}=33.71, p<0.0001$; genotype, $F_{(1,34)}=4.126, p=0.0501$; interaction $\left.F_{(1,34)}=3.398, p=0.074\right)$ followed by Tukey test: $\mathrm{EAE}$ WT vs EAE KO, ${ }^{*} p=0.0114$; healthy WT vs EAE WT, ${ }^{* * * *} p \leq 0.0001$. C, Two-way ANOVA (healthy/EAE, $F_{(1,17)}=6.729, p=0.0189 ;$ genotype, $F_{(1,17)}=2.378, p=0.14415$; interaction $\left.F_{(1,17)}=1.862, p=0.1902\right)$ followed by Tukey test: healthy WT vs EAEWT, ${ }^{*} p=0.03$; EAEWT vs EAE KO ${ }^{*} p=0.04$. D, Two-way ANOVA (health/EAE, $F_{(1,35)}=41.39, p<0.0001$; genotype, $F_{(1,35)}=2.936, p=0.0955$; interaction $\left.F_{(1,35)}=1.912, p=0.1755\right)$ followed by Tukey test: healthy KO vs EAE KO, ${ }^{* *} p=0.0061$; healthy WT vs EAE WT, ${ }^{* * *} p \leq 0.0001$. $E$, Two-way ANOVA (interaction $F_{(1,17)}=3.898, p=0.0648$; healthy/EAE, $F_{(1,17)}=15.07, p=0.0012$; genotype, $F_{(1,17)}=0.9879, p=0.3342$ ) followed by Tukey post hoc test: EAE WT vs EAE K0, ${ }^{*} p=0.0306$; healthy WT vs EAEWT, ${ }^{* *} p=0.0016$. $F$, Two-way ANOVA (healthy/EAE, $F_{(1,35)}=$ $0.1962, p=0.6605$; genotype, $F_{(1,35)}=0.9648, p=0.3327$; interaction $F_{(1,35)}=0.3970$, $p=0.5327)$ followed by Tukey test: healthy WT vs EAEWT, $p=0.4490$; EAEWT vs EAE KO, $p=$ 0.2167. $\boldsymbol{H}$, Two-way ANOVA (health/EAE, $F_{(1,37)}=15.18, p=0.0004$; genotype, $F_{(1,37)}=$ $2.613, p=0.1145$; interaction $\left.F_{(1,37)}=2.666, p=0.1110\right)$ followed by Tukey test: EAE WT vs EAE KO ${ }^{*} p=0.0237$; healthy WT vs EAE WT, ${ }^{* *} p=0.002$. I, Two-way ANOVA (health/EAE, $F_{(1,37)}=9.911, p=0.0032$; genotype, $F_{(1,37)}=2.244, p=0.1426$; interaction $F_{(1,37)}=$ $1.210, p=0.2783)$ followed by Tukey test: EAE WT vs EAE KO ${ }^{*} p=0.0267$, healthy WT vs EAE $W T,{ }^{*} p=0.0227$. Graphs represent mean \pm SEM. top of MBMECs. The lower compartments of the transwell chambers were filled with transmigration media, containing SDF-1 $(100 \mathrm{ng} / \mathrm{ml})$ as a chemoattractant. After 1 and $3.5 \mathrm{~h}$, cells from the lower compartment of the transwell chamber were harvested and counted using CASY.

In vitro static adhesion assay. A total of $4 \times 10^{4} \mathrm{MBMECs} /$ well of gelatin-fibronectin coated 96-well plate were grown for $4-5 \mathrm{~d}$ and stimulated $16 \mathrm{~h}$ before the assay with $5 \mathrm{~nm}$ TNF $\alpha$. OTII-OVA T cells were stimulated for $2 \mathrm{~d}$ with $1.5 \%$ IL-2, labeled with $2 \mu \mathrm{M}$ Cell Tracker Green CMFDA (Invitrogen), and added $1 \times 10^{5}$ cells/well on top of MBMECs for $30 \mathrm{~min}$. Next, wells were washed 5 times with PBS containing $1 \mathrm{~mm}$ each of $\mathrm{CaCl}_{2}$ and $\mathrm{MgCl}_{2}$, fixed with $4 \% \mathrm{PFA}$, and analyzed under an inverted epifluorescent microscope (Carl Zeiss Axiovert $200 \mathrm{~m}$ ). The results were quantified with ImageJ $1.48 \mathrm{v}$.

In vitro adhesion assay under flow. A total of $8 \times 10^{4} \mathrm{MBMECs} / \mathrm{chan}-$ nel, of the six-channel $\mu$-Slide VI ${ }^{0.4}$ (ibidi) coated with gelatin and fibronectin (as above), were grown for 3-4 $\mathrm{d}$ and stimulated $16 \mathrm{~h}$ before the assay with $5 \mathrm{~nm} \mathrm{TNF} \alpha$. OTII-OVA T cells were stimulated for $2 \mathrm{~d}$ with $1.5 \%$ IL-2 and labeled with $2 \mu \mathrm{M}$ Cell Tracker Green CMFDA. Labeled T cells were resuspended at $2.5 \times 10^{5} / \mathrm{ml}(20 \mathrm{ml}$ per flow) in HBSS with 1 mu each of $\mathrm{CaCl}_{2} / \mathrm{MgCl}_{2} 1.2 \%$ BSA and $25 \mathrm{~mm}$ HEPES, mixed with SDF1 $(50 \mathrm{ng} / \mathrm{ml})$ and added to the MBMEC monolayer at 0 to 2 dyne $/ \mathrm{cm}^{2}$ in 20 min. Videos were recorded under a $10 \times$ objective and analyzed with TrackMate 20.

Immunohistochemistry. The brains were isolated at peak EAE (p.i. day 17-20), embedded in O.C.T. Compound (Tissue-Tek), snap-frozen, and sectioned on a cryotome from dorsal to ventral. For staining, $6 \mu \mathrm{m}$ sections from 4 different regions were taken. Sections were air-dried for $30 \mathrm{~min}$, fixed for $15 \mathrm{~min}$ with cold methanol at $-20^{\circ} \mathrm{C}$, washed twice with PBS, and blocked with $1 \%$ BSA/10\% goat serum in PBS for $1 \mathrm{~h}$. Incubation with primary anti-pan-laminin and anti-CD45 antibodies was performed overnight at $4^{\circ} \mathrm{C}$. Bound antibodies were visualized using AlexaFluor-488-conjugated donkey anti-rabbit and AlexaFluor-568conjugated donkey anti-rat secondary antibodies, which were applied for $1 \mathrm{~h}$. Sections were mounted with Dako Fluorescence Mounting Medium, and the number of inflammatory cuffs/section was counted using a Carl Zeiss Axioscope 2.

For brain vessel staining, the brains were isolated, fixed for $4 \mathrm{~h}$ with $4 \%$ PFA at $4^{\circ} \mathrm{C}$, embedded in $4 \%$ low melting agarose, and stored at $4^{\circ} \mathrm{C}$. Thick sections of $100 \mu \mathrm{m}$ were cut on a vibratome, permeabilized with $0.3 \%$ Triton X-100/0.1\% Tween 20 in PBS for $1 \mathrm{~h}$ at $4^{\circ} \mathrm{C}$, and blocked with $4 \%$ BSA $/ 0.3 \%$ Triton X-100 in PBS for $1 \mathrm{~h}$ at $4^{\circ} \mathrm{C}$. Subsequent incubation with primary and secondary antibodies (AlexaFluor-488conjugated donkey anti-rabbit) was performed overnight at $4^{\circ} \mathrm{C}$. Slides were mounted with Dako Fluorescence Mounting Medium and examined with a confocal microscope (Carl Zeiss LSM 880), and analyzed using ImageJ $1.48 \mathrm{v}$.

Spinal cord histological analysis. For evaluation of immune-mediated inflammation in the spinal cord, spinal cords were fixed in 4\% PFA for $2 \mathrm{~d}$ at $4^{\circ} \mathrm{C}$, rehydrated in $15 \%$ and $30 \%$ glucose for $3 \mathrm{~d}$ each, snap-frozen in OCT compound, and sectioned on a cryotome. Ten-micrometer sections from the lower lumbar part of the spinal cord were stained for Hematoxylin and Eosin (H\&E) and Luxol Fast Blue (LFB) (Solvent blue 38 , Sigma Millipore) to evaluate the number of inflammatory foci and the degree of demyelination, respectively. Sections were analyzed with an inverted epifluorescent microscope (Carl Zeiss Axiovert 200) and data quantified with ImageJ $1.48 \mathrm{v}$.

$B B B$ permeability in vivo. For analysis of $\mathrm{BBB}$ permeability during EAE development, $100 \mu \mathrm{l}$ of $2 \%$ Evans blue in PBS was injected intravenously into the tail vein; mice were perfused with PBS 1 or $24 \mathrm{~h}$ after injection, and brains and spinal cords were removed. Tissues were placed in formamide for $5 \mathrm{~d}$, and the concentration of Evans blue was measured at 620 $\mathrm{nm}$ (Shimadzu). Kidney tissue from naive mice treated in the same manner served as positive controls. The amount of tracer in the tissues was determined from the formula A620/wet weight (g).

Isolation of MBMECs. MBMECs were obtained as described previously (Ruck et al., 2014). Briefly, brains from 4 or 5 mice (10- to14-week-old) were isolated. The forebrains, without meninges, were transferred to a tube with $13.5 \mathrm{ml}$ DMEM. The tissue was minced first with a plastic $25 \mathrm{ml}$ pipette and then using a $10 \mathrm{ml}$ pipette. The suspension was further di- 
A
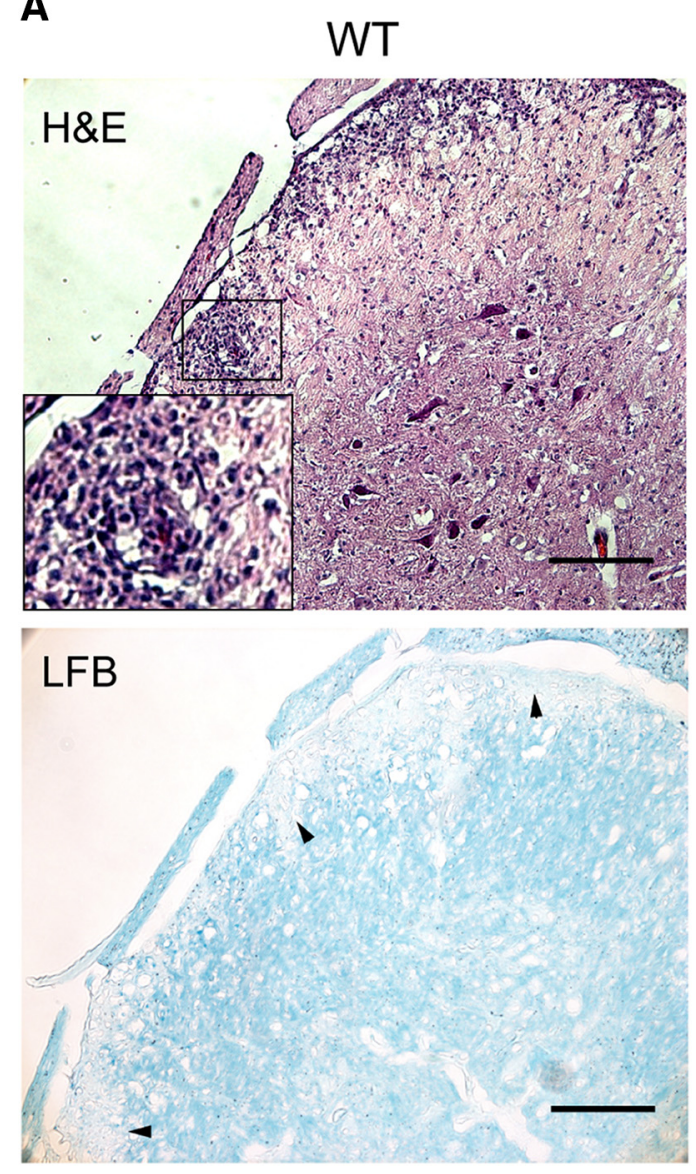

$\mathrm{Cttn}^{-1-}$
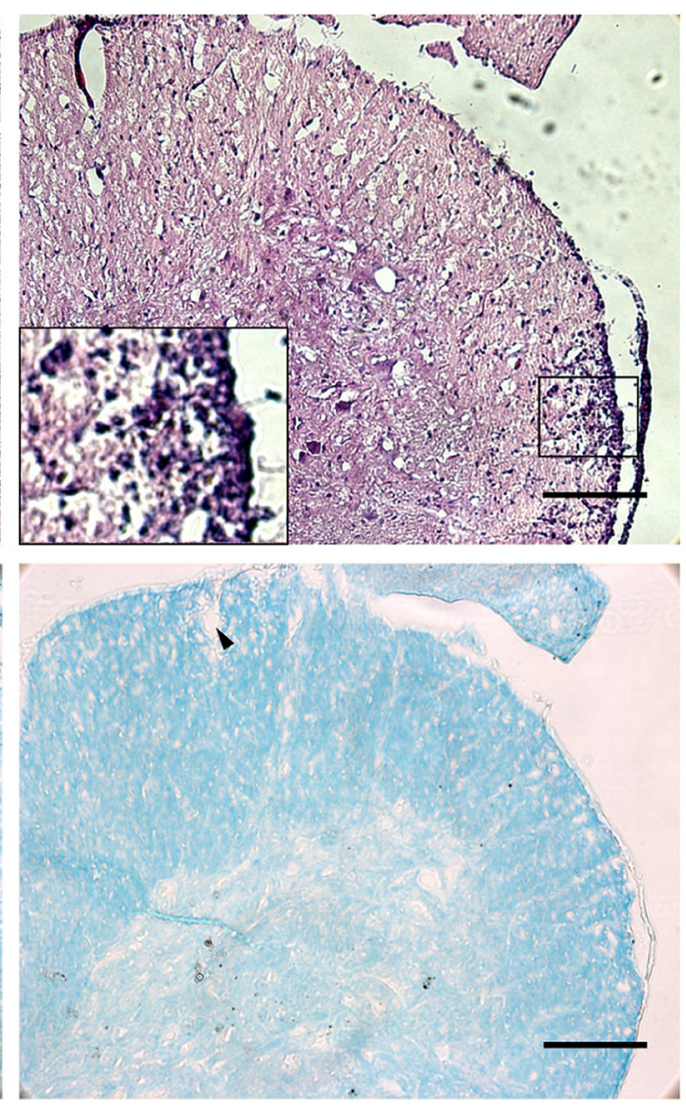

B

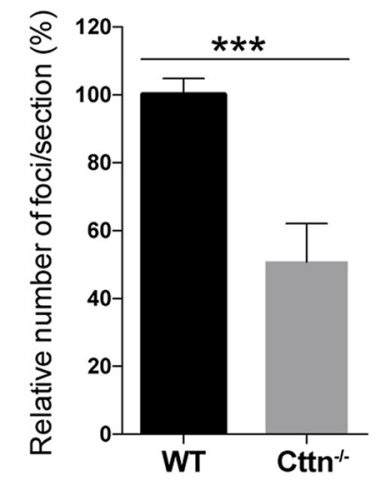

Figure 4. Leukocyte infiltration and demyelination in the spinal cord is reduced in $\mathrm{Cttn}^{-1-}$ mice during EAE. WT or $\mathrm{Cttn}^{-1-}$ mice were killed when WT mice reached peak EAE. The lumbar parts of the spinal cords were isolated, sectioned, and examined for inflammatory foci and for axonal demyelination by H\&E and LFB staining, respectively. $A$, Representative staining of inflammatory foci (insets, higher magnifications of marked areas) and demyelination (arrowheads indicate areas of demyelination). $\boldsymbol{B}$, Quantification of the number of inflammatory foci (with WT mice at peak EAE set as 100\%) in 4 random sections per mouse. Graph represents mean \pm SEM of three independent experiments with $n=4, n=5$, and $n=6$ WT mice (total $n=15$ ) and $n=4$ /experiment (total $n=12) \mathrm{Cttn}^{-1-}$ mice $\left.{ }^{* * *} p=0.0002\right)$. C, The area of demyelination in 3 random sections/mouse. Graph represents mean \pm SEM of four independent experiments with $n=4, n=4, n=6$, and $n=6$ (total $n=20)$ WT mice and $n=4, n=4, n=5$, and $n=6$ (total $n=19)\left(\mathrm{Ctn}^{-1-}\right.$ mice $\left(^{* * *} p=0.0001\right)$. Scale bar, $150 \mu$ m. Statistics were analyzed with unpaired two-tailed Student's $t$ test.

gested with $0.6 \mathrm{ml} 10 \mathrm{mg} / \mathrm{ml}$ collagenase and $20 \mu \mathrm{l} 10 \mathrm{mg} / \mathrm{ml}$ DNase in DMEM for $1 \mathrm{~h}$ at $37^{\circ} \mathrm{C}$. Then $10 \mathrm{ml}$ DMEM was added and cells were centrifuged at $1400 \mathrm{rpm}$ for $20 \mathrm{~min}$ at $4^{\circ} \mathrm{C}$. To remove the myelin, the pellet was resuspended in $25 \mathrm{ml} 20 \%$ BSA-DMEM and centrifuged at $1500 \mathrm{rpm}$ for $30 \mathrm{~min}$ at $4^{\circ} \mathrm{C}$. The pellet was resuspended in $10 \mathrm{ml}$ DMEM and further digested with $0.3 \mathrm{ml}$ collagenase ( $10 \mathrm{mg} / \mathrm{ml}$ in DMEM), $0.3 \mathrm{ml}$ dispase (10 $\mathrm{U} / \mathrm{ml}$ ), and $10 \mu \mathrm{l}$ DNase for $1 \mathrm{~h}$ at $37^{\circ} \mathrm{C} ; 10 \mathrm{ml}$ DMEM was then added, and the cells were centrifuged at $1400 \mathrm{rpm}$ for $20 \mathrm{~min}$ at $4^{\circ} \mathrm{C}$. The cells were resuspended in $2 \mathrm{ml}$ endothelial cell media and seeded onto 12-well plates, coated with $40 \%$ gelatin and $0.1 \mathrm{mg} / \mathrm{ml}$ fibronectin (Sigma Millipore).

Immunocytochemistry. For staining, a total of $5 \times 10^{4} \mathrm{MBMECs} /$ well of gelatin-fibronectin coated (as above), 8 -well chamber slides (ibidi) were grown for $4-5 \mathrm{~d}$. Cells were washed twice with PBS, permeabilized with $0.5 \%$ Tween 20 for $10 \mathrm{~min}$, and fixed with $4 \%$ PFA for $5 \mathrm{~min}$ at room temperature. Nonspecific binding was blocked with 3\%BSA/PBS, pH 7.4 , for $1 \mathrm{~h}$ at room temperature. Subsequently, cells were incubated for overnight at $4^{\circ} \mathrm{C}$ with anti-cortactin, anti-occludin, anti-VE-cadherin, anti-ZO-1, or anti-ICAM-1 primary antibodies diluted in PBS containing $2 \%$ BSA, followed by incubation with fluorescently conjugated secondary antibodies (Invitrogen). The cells were washed twice with PBS, stained with $2 \mu \mathrm{g} / \mathrm{ml} \mathrm{Hoechst} 33342$ (Invitrogen) for $5 \mathrm{~min}$, and kept in $0.01 \% \mathrm{NaN}_{3} / \mathrm{PBS}$. Staining was analyzed a confocal microscope (Carl Zeiss LSM 880) and data analyzed with ImageJ $1.48 \mathrm{v}$ processing software.

Experimental design and statistical analysis. Mice were male or female, and the experimental groups were gender- and age-matched. For all experiments, the number of mice used and the number of independent experiments are stated in the figure legends. All data values are presented as the mean \pm SEM. Statistical analysis was performed using GraphPad Prism 6. Distribution of data was evaluated for normality using D'Agostino and Pearson omnibus test. The significance between two groups was evaluated using the unpaired Student's $t$ test or by Mann-Whitney Rank Sum Test. Comparison between more than two groups was analyzed using one-way or two-way ANOVA followed by Tukey post hoc test. ANOVA was presented as $F_{(x, y)}$, where $x$ is degree of freedom from between the sample groups and $y$ is degree of freedom from within the samples.

\section{Results}

\section{Cortactin deficiency results in reduced EAE severity}

To examine the role of cortactin in neuroinflammation and leukocyte extravasation across the $\mathrm{BBB}$, we analyzed the development of active EAE in cortactin gene-inactivated $\left(\mathrm{Cttn}^{-1-}\right)$ mice. To this end, we induced active EAE in $\mathrm{Cttn}^{-1-}$ and WT littermates by immunization with the $\mathrm{MOG}_{35-55}$ peptide. EAE clinical symptoms developed in the form of gradual paralysis, peaking between day 16 and 20 p.i. (Fig. $1 A$ ). Evaluation of the clinical symptoms showed that the overall severity of disease in $\mathrm{Cttn}^{-1-}$ mice was significantly lower than in WT mice (Fig. $1 A-C$ ), with no difference in the day of disease onset (Fig. 1D). The percentage of mice with severe clinical scores was 3 times higher among WT 


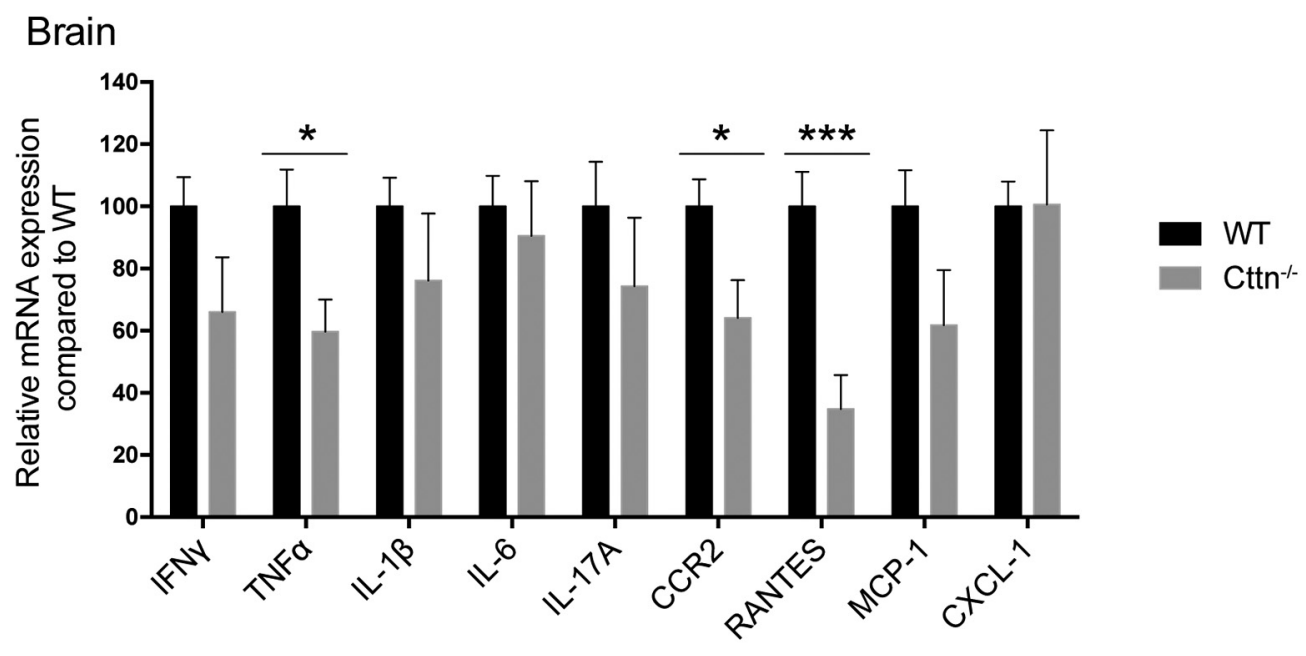

Spinal cord

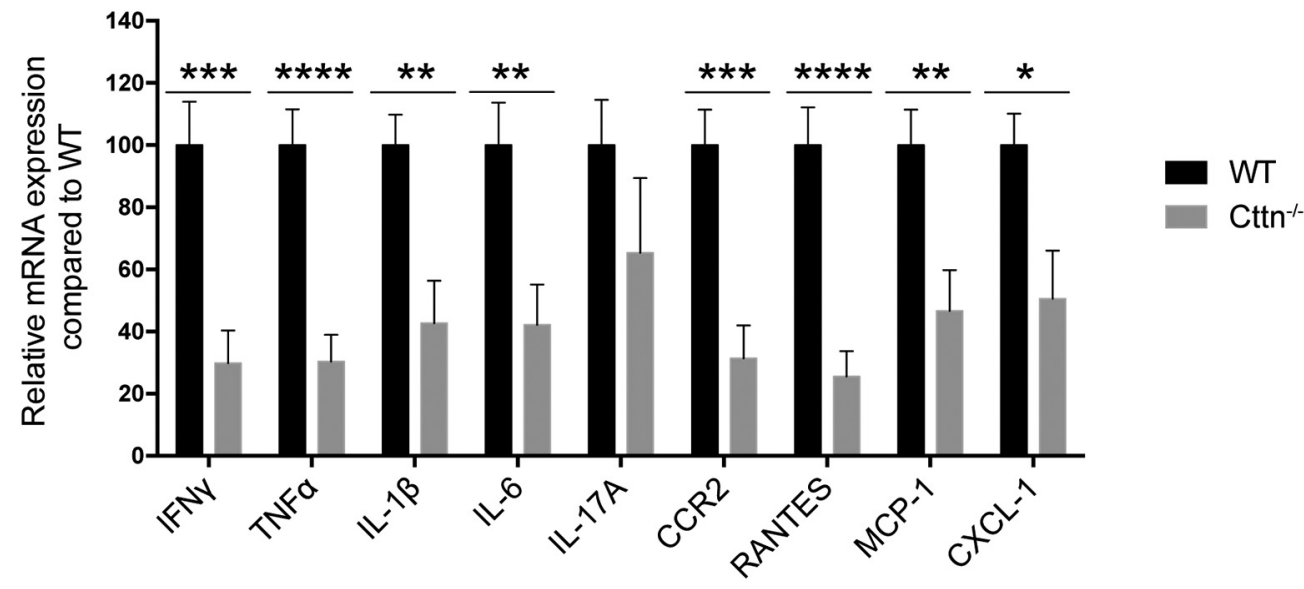

Figure 5. Lack of cortactin leads to lower levels of proinflammatory cytokines in the inflamed CNS. WT or (ttn ${ }^{-1-}$ mice were killed at peak EAE, and total RNA from brains and spinal cords were isolated. Cytokine expression in brain (top) and spinal cord (bottom) was measured by qRT-PCR. mRNA expression was normalized to GAPDH, and gene expression in WT mice was set as 100\%. Graphs represent mean \pm SEM of three independent experiments with $n=3, n=5$, and $n=6$ (total $n=14$ ) for EAE WT mice and EAE ttnn $^{-1-}$ mice. Statistics were analyzed with unpaired two-tailed Student's t test (brain: IFN $\gamma$, TNF- $\alpha$, IL-6, MCP-1, CCR2; spinal cord: TNF- $\alpha$, IL-6, MCP-1, RANTES, IL-17), or with Mann-Whitney U test (brain: IL-1 $\beta$, RANTES, IL-17, CXCL1; spinal cord: IFN $\gamma$, IL-1 $\beta$, CCR2, CXCL1). Brain: ${ }^{*} p=0.0251$ (CCR2), ${ }^{*} p=0.0165$ (TNF- $\left.\alpha\right),{ }^{* * *} p=0.0003$ (RANTES). Spinal cord: ${ }^{*} p=0.0138\left(\right.$ (CXCL1), ${ }^{* *} p=0.002$ (IL-1 $\left.\beta\right),{ }^{* *} p=0.005$ (IL-6, MCP-1), ${ }^{* * *} p=0.0006($ IFN $\gamma),{ }^{* * *} p=0.0002\left(\right.$ CCR2), ${ }^{* * * *} p \leq 0.0001$ (TNF- $\alpha$, RANTES).

mice (84.2\%) compared with $\mathrm{Cttn}^{-1-}$ animals (26.3\%), and the majority of the KO mice (57.9\%) developed mild clinical symptoms, compared with $15.8 \%$ of WT mice (Fig. $1 E$ ). Together, these data demonstrate that the absence of cortactin results in reduced EAE severity.

Immune response to $\mathrm{MOG}_{35-55}$ peptide is not affected by lack of cortactin

When bred under pathogen-free conditions, healthy $\mathrm{Cttn}^{-1-}$ mice developed normal without any visual defects and had normal peripheral leukocyte, platelet, and erythrocyte counts similar to control animals (Schnoor et al., 2011). Since EAE commences with induction of a peripheral immune response, we tested whether cortactin gene inactivation could affect this process. To this end, we analyzed the proportions and numbers of CD4 ${ }^{+} \mathrm{T}$ cells and $\mathrm{CD} 11 \mathrm{~b}^{+}$macrophages in the blood, lymph nodes, and spleens of $\mathrm{Cttn}^{-1-}$ and WT animals before EAE onset (day 10 p.i.) (Fig. $2 A$ ) and at the peak disease (Fig. $2 B$ ), revealing no differences between $\mathrm{Cttn}^{-1-}$ and WT animals.

As another approach to test a potential role of cortactin in peripheral immune responses, we performed in vitro BrdU col- orimetric proliferation assays to compare the proliferation of WT and $\mathrm{Cttn}^{-1-}$ splenocytes, isolated on day 10 p.i. and restimulated in vitro with different concentrations of $\mathrm{MOG}_{35-55}$ peptide. A larger proportion of splenocytes in both WT and $\mathrm{Cttn}^{-1-} \mathrm{EAE}$ mice showed active proliferation compared with healthy animals (non-EAE), indicating the efficiency of in vivo splenocyte priming toward the myelin antigen. In vitro restimulation with $\mathrm{MOG}_{35-55}$ peptide further increased the proportion of proliferating splenocytes in EAE animals. In all conditions, we could not detect differences in splenocyte proliferation of WT and $\mathrm{Cttn}^{-1-}$ mice (Fig. 2C), consistent with our in vivo data. Last, we detected no differences in IL-17A and IFN $\gamma$ expression by MOGreactivated splenocytes (Fig. $2 D$ ). Together, the absence of cortactin does not interfere with the induction of the immune response.

\section{Cortactin deficiency restricts leukocyte infiltration and inflammation in the CNS}

To test whether a decrease in EAE scores in the absence of cortactin was caused by reduced leukocyte recruitment into the brain parenchyma, we isolated immune cells from the brain of WT and 
A

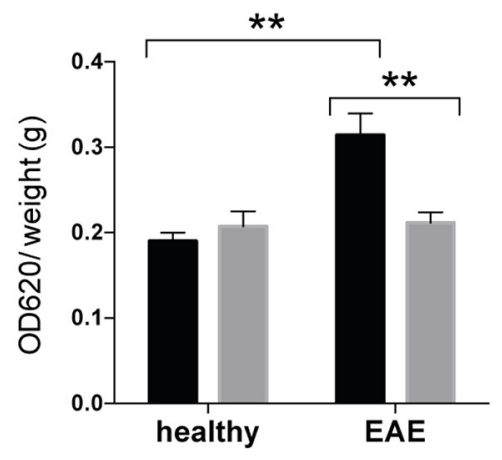

D

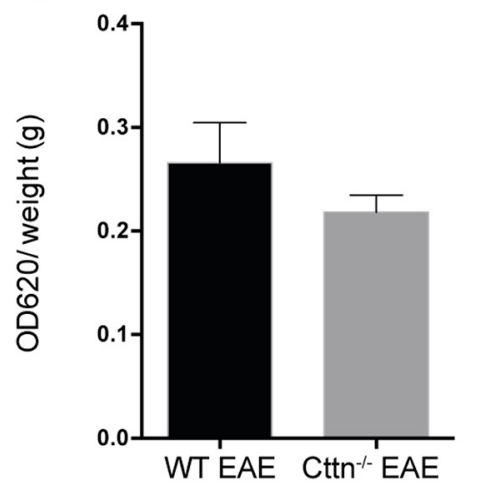

$\mathbf{F}$

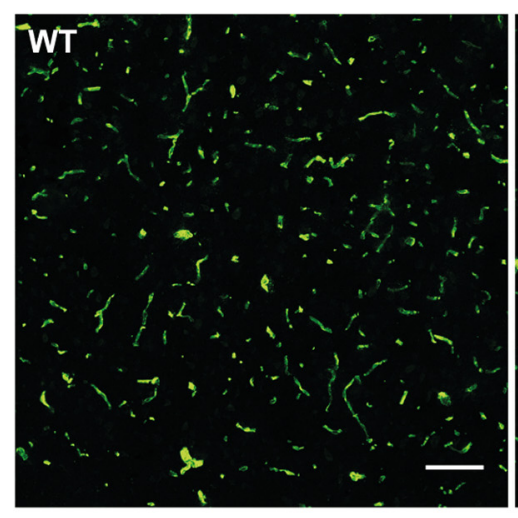

B

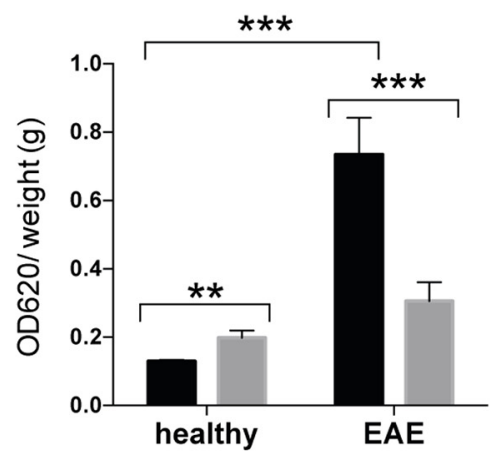

E
C

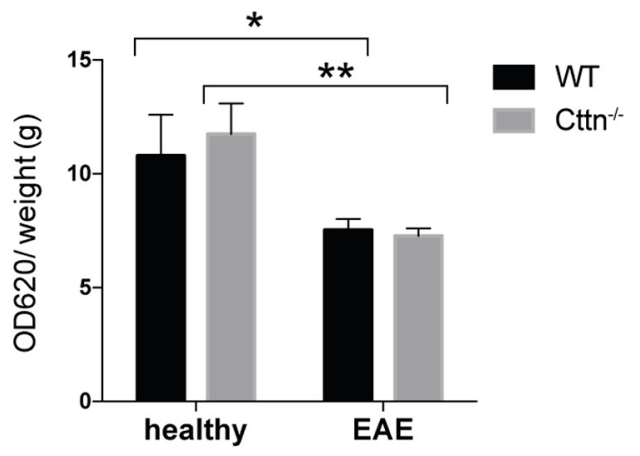

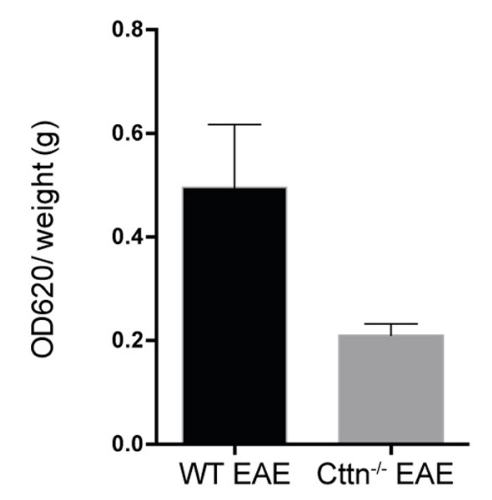

G
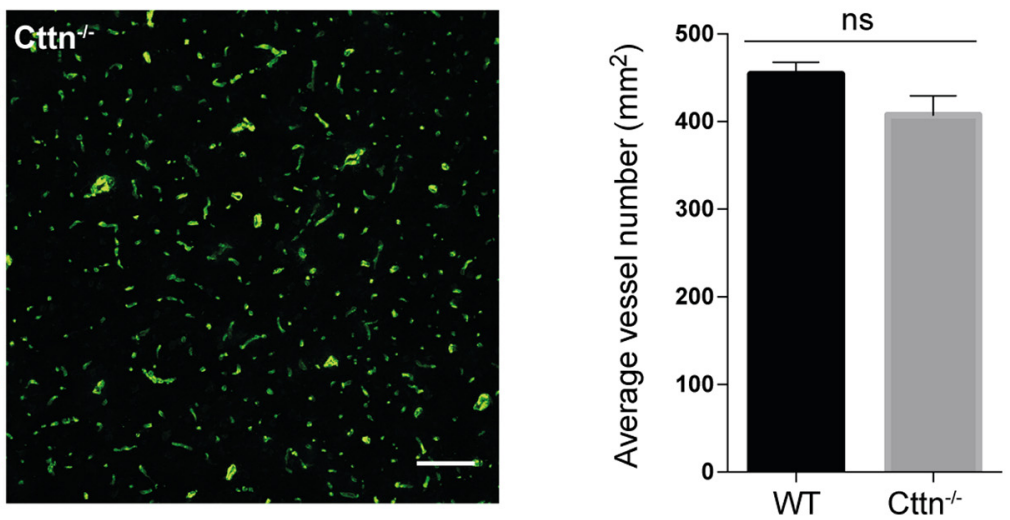

Figure 6. Lack of cortactin results in reduced vascular permeability in the inflamed CNS. Healthy and EAE-subjected WT or $\mathrm{Cttn}^{-1-}$ mice were injected intravenously with Evans blue on day 10 p.i. and killed $24 \mathrm{~h}$ later. Animals were perfused with PBS, and brains $(\boldsymbol{A})$, spinal cords $(\boldsymbol{B})$, and kidneys $(\boldsymbol{C})$ were isolated. The dye was extracted from tissue and measured at $620 \mathrm{~nm}$; data are expressed relative to organ weight. Graphs represent mean \pm SEM of three independent experiments with $n=3, n=5$ (total $n=8 /$ group) for healthy WT or Cttn ${ }^{-1-}$ mice; $n=5, n=6$, and $n=6$ (total $n=17$ ) for EAE-subjected WT mice and with $n=4, n=6$, and $n=6$ (total $n=16$ ) for EAE-subjected $\left(t+n^{-1-}\right.$ mice. D, E, Similar to $A$, B, except that brain and spinal cord permeability was measured on the peak of EAE (day 17 p.i.), and animals were killed $1 \mathrm{~h}$ after Evans blue injections. Graphs represent mean \pm SEM of experiment with $n=4 /$ group for WT or $\left(t t n{ }^{-1-}\right.$ mice. $F$, Representative cryosections of brains from WT mice and $\mathrm{Cttn}^{-1-}$ mice on the peak of EAE, stained with anti-pan-laminin antibodies. Scale bar, $100 \mu \mathrm{m}$. G, Average vessel density was measured in 5 different areas/animal with $n=5$ mice/group for WT and $C t t^{-1-}$. Statistics were analyzed as follows: $A$, two-way ANOVA (healthy/EAE, $F_{(1,44)}=8.324, p=0.006 ;$ genotype, $F_{(1,44)}=3.704$, $p=0.0608$; interaction $\left.F_{(1,44)}=7.274, p=0.0099\right)$ followed by Tukey test: EAEWT vsEAEKO, ${ }^{* *} p=0.0014$; healthy WT vs EAE wt; ${ }^{* *} p=0.002 . B$, Two-way ANOVA (healthy/EAE, $F_{(1,44)}=15.60$, $p=0.0003$; genotype, $F_{(1,44)}=4.006, p=0.0515$; interaction $\left.F_{(1,44)}=7.648, p=0.0083\right)$ followed by Tukey test: healthy WT vs healthy $K 0,{ }^{* *} p=0.002$; healthy WT vs EAEWT, ${ }^{* * *} p=0.0001$; EAE WT vs EAE KO, ${ }^{* * *} p=0.0009$. C, Two-way ANOVA (healthy/EAE, $F_{(1,44)}=19.14, p<0.0001 ;$ genotype, $F_{(1,44)}=0.1582, p=0.6927$; interaction $\left.F_{(1,44)}=0.4692, p=0.4969\right)$ followed by Tukey test: healthy WT vs EAE WT, ${ }^{*} p=0.00382$; healthy KO vs EAE KO ${ }^{* *} p=0.0074 . \boldsymbol{D}, \boldsymbol{E}, \boldsymbol{G}$, Statistics were analyzed with unpaired two-tailed Student's $t$ test: $\boldsymbol{D}, p=0.3244 ; \boldsymbol{E}, p=0.097 ; \boldsymbol{G}$, not significant (ns) $p=0.12$. Graph represents mean \pm SEM

$\mathrm{Cttn}^{-1-}$ mice at a time point when WT mice reached the peak clinical score of EAE. The total number of isolated leukocytes was determined by a cell counter and subsequently quantified by flow cytometry for the presence of CD $45^{\text {high }}$ leukocytes, CD ${ }^{+}$T cells, $\mathrm{CD}^{+} \mathrm{T}$ cells, CD11b ${ }^{+}$macrophages, and Ly6G neutrophils. Our results showed that cortactin deficiency resulted in a significant $45 \%$ decrease in the proportion of CD $45^{\text {high }}$ immune cells
(Fig. 3A) and in the different leukocyte populations that had infiltrated into the inflamed brains: $\mathrm{CD}^{+}{ }^{+} \mathrm{T}$ cells ( $49 \%$ reduced), $\mathrm{CD}^{+}{ }^{+} \mathrm{T}$ cells $\left(50 \%\right.$ reduced), $\mathrm{CD} 11 \mathrm{~b}{ }^{+}$macrophages $(33 \%$ reduced), and Ly6G neutrophils (44\% reduced) (Fig. 3B-E). At the same time, there was no difference in the numbers of $\mathrm{CD} 11 \mathrm{~b}{ }^{+} \mathrm{CD} 45^{\text {low }}$ microglia in $\mathrm{KO}$ and WT brains (Fig. $3 F$ ). Similar results were obtained for the spinal cord, with a decrease in 
CD $45^{\text {high }}$ cells (46\% reduced), $\mathrm{CD}^{+}{ }^{+} \mathrm{T}$ cells (50\% reduced), and CD $11 \mathrm{~b}^{+}$macrophages (40\% reduced) in $\mathrm{Cttn}^{-1-}$ compared with WT mice (Fig. $3 G-I$ ). A more detailed analysis of CD4 T-cell subsets revealed that, at peak of disease, cortactin deficiency caused a strong reduction of Th17 cells, but not Th1 cells, in spinal cord (Fig. $3 \mathrm{~K}$ ), whereas no changes were found for both T-cell subtypes in brain (Fig. $3 J$ ) and spleen (Fig. 3L).

As an additional criterion for the efficiency of leukocyte infiltration into the CNS, we used immunohistochemistry to analyze the presence of inflammatory cuffs in the brains of $\mathrm{Cttn}^{-1-}$ and WT mice. Inflammatory cuffs are typical structures that are observed in EAE, formed due to the accumulation of immune cells between endothelial and parenchymal basement membranes of the BBB (Sixt et al., 2001). These structures were visualized at the peak of EAE by staining for the two basement membranes using pan-laminin antibody and with antiCD45 to mark leukocytes (Fig. 3M). The number of cuffs were counted in four different brain sections (cutting intervals 240 $\mu \mathrm{m})$ per animal and the average number of cuffs per section was calculated. Quantification showed a significant $48 \%$ decrease in the number of proinflammatory cuffs in $\mathrm{Cttn}^{-1-}$ mice compared with their WT counterparts (Fig. $3 N$ ). The average size of cuffs was unaffected by cortactin gene deficiency (Fig. 3O).

To evaluate the degree of inflammation in the spinal cord at peak EAE, we performed H\&E and LFB staining of frozen, transverse sections taken from the spinal cord lumbar region to visualize leukocyte infiltration and neurodegeneration (demyelination), respectively. The severity of leukocyte infiltration was quantified by determining the average number of inflammatory foci per section, using 4 sections per mouse. As shown in Figure $4 A, B, \mathrm{Ctnn}^{-1-}$ animals had a significant reduction in the number of inflammatory foci compared with WT mice. Neurodegeneration in the spinal cord was assessed by LFB staining; absence of staining defines sites of demyelination and quantified as average demyelinated area per section from 3 different sections per animal; data were expressed relative to the demyelination observed in WT mice. This revealed that $\mathrm{Cttn}^{-1-}$ mice exhibited $51 \%$ less myelin loss than WT animals (Fig. 4A, C). These data collectively suggest that cortactin is important for the process of immune cell diapedesis into the CNS, thereby supporting the progression of pathological changes and the development of neuroinflammation during EAE.

\section{Cortactin-deficient mice showed reduced expression of proinflammatory cytokines}

We next tested whether the absence of cortactin affected expression of proinflammatory mediators in the inflamed CNS. To this end, we isolated RNA from the brains and spinal cords of WT and $\mathrm{Cttn}^{-1-}$ mice at peak EAE and performed qRT-PCR. Several proinflammatory cytokines, chemokines, and chemokine receptors were analyzed, revealing generally lower levels of production of proinflammatory mediators in the brains of $\mathrm{Cttn}^{-/-}$mice compared with WT mice (Fig. 5). The most profound difference was observed for RANTES, a chemokine for T cells and monocytes. A significant decrease in TNF $\alpha$ and CCR2, but not IFN $\gamma$,
IL-1 $\beta$, IL-6, IL-17A, MCP-1, and CXCL1, expression was measured in $\mathrm{Cttn}^{-1-}$ compared with WT brains. In the inflamed spinal cord, most analyzed cytokines and chemokine receptors analyzed were expressed at significantly lower levels in $\mathrm{Cttn}^{-1-}$ mice compared with WT mice, including TNF $\alpha$, IFN $\gamma$, IL- $1 \beta$, IL-6, CCR2, RANTES, MCP-1, and CXCL1 (Fig. 5). IL-17A was also reduced, but not at a statistically significant level. The reduced levels of proinflammatory cytokines in the absence of cortactin is consistent with the decreased leukocyte infiltration observed in these mice and probably contributes to the lower EAE scores in $\mathrm{Cttn}^{-/-}$animals.

\section{Cortactin-deficient mice have reduced vascular permeability in the CNS}

We previously showed that cortactin gene inactivation causes a significant increase in the basal and histamine-induced vascular permeability in the skin (Schnoor et al., 2011). We therefore tested whether the absence of cortactin has similar effects on vascular permeability in the CNS during EAE. EAE-subjected WT and $\mathrm{Cttn}^{-1-}$ mice were injected at day 10 i.p. with Evans blue and leakage into tissues was measured $24 \mathrm{~h}$ later; nonimmunized, healthy WT and $\mathrm{Cttn}^{-1-}$ mice served as controls. In general, increased vascular permeability was measured in the brain and spinal cords of WT EAE mice compared with healthy WT and $\mathrm{Cttn}^{-1-}$ controls (Fig. 6A,B). However, as shown in Figure $6 B$, healthy $\mathrm{Cttn}^{-1-}$ mice showed an increase in the basal permeability of blood vessels in the spinal cord, consistent with our previous data from the skin, and little or no increase in permeability during EAE. Comparison of the MOG-injected animals revealed significant $30 \%$ and $58 \%$ reductions in Evans blue leakage into the brain (Fig. 6A) and the spinal cord (Fig. 6B) of KO mice compared with WT. Vascular permeability in the kidney of the same mice, which served as control, was not affected by cortactin gene inactivation (Fig. 6C). Similar experiments were performed at peak of clinical score, with Evans blue being injected for only $1 \mathrm{~h}$ before measuring leakage. We detected a significant reduction in permeability in the spinal cord of $\mathrm{Cttn}^{-1-}$ mice (Fig. 6E), whereas different from the onset of EAE, brain permeability at the peak of disease was similar in WT and $\mathrm{Cttn}^{-1-}$ mice (Fig. 6D). Vessel density in the brain was similar in WT and $\mathrm{Cttn}^{-1-}$ mice (Fig. 6F, G). Collectively, our results suggest that cortactin gene 
A
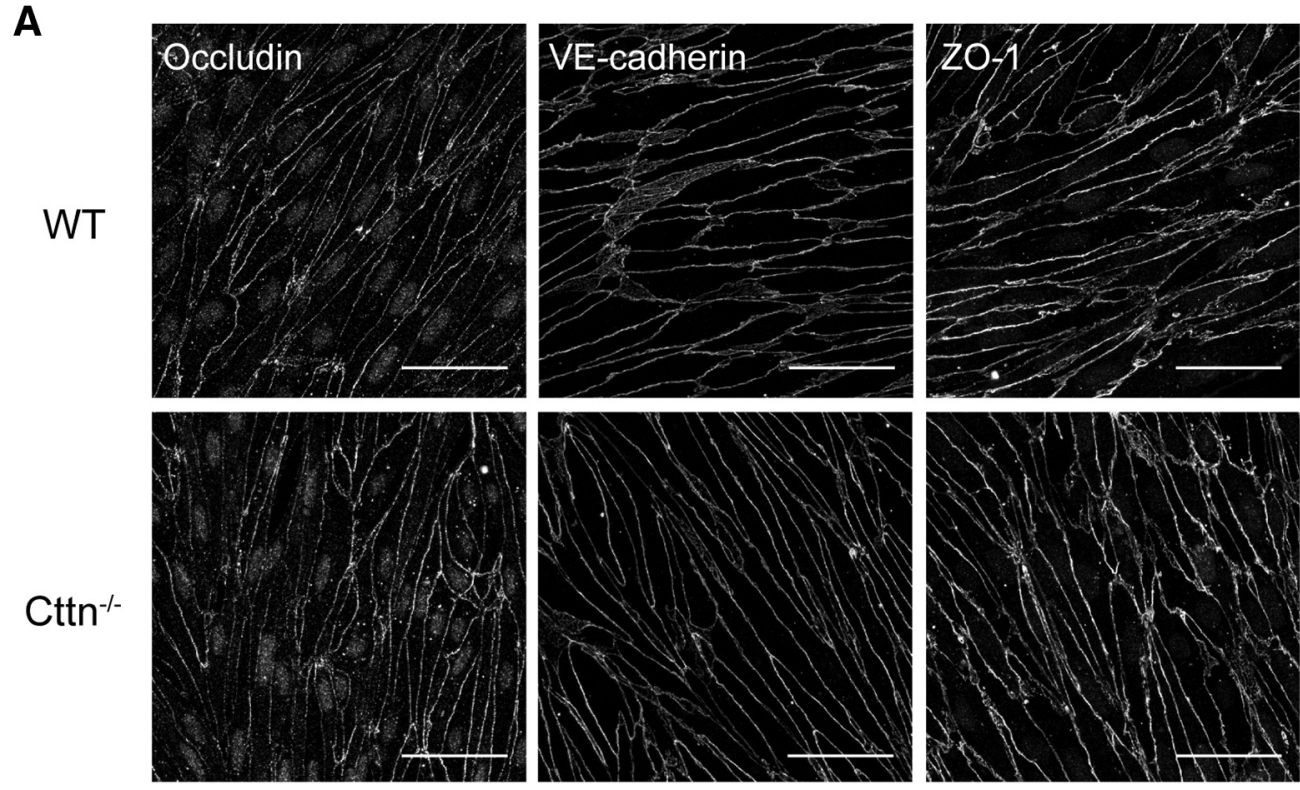

B

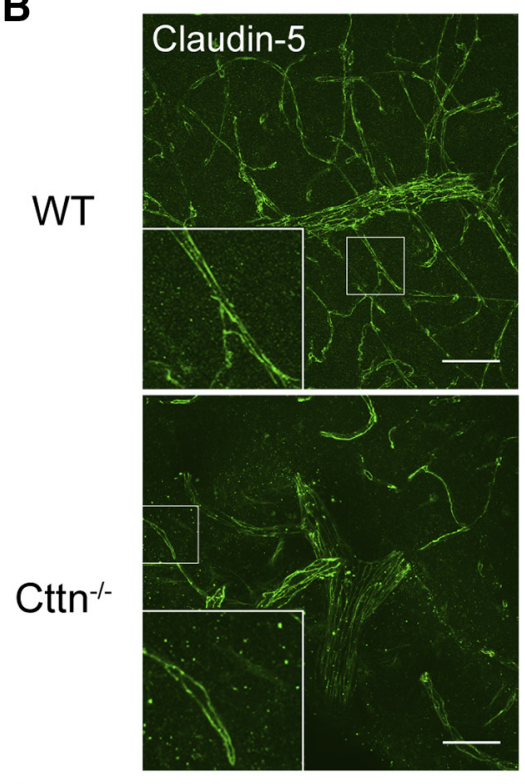

C
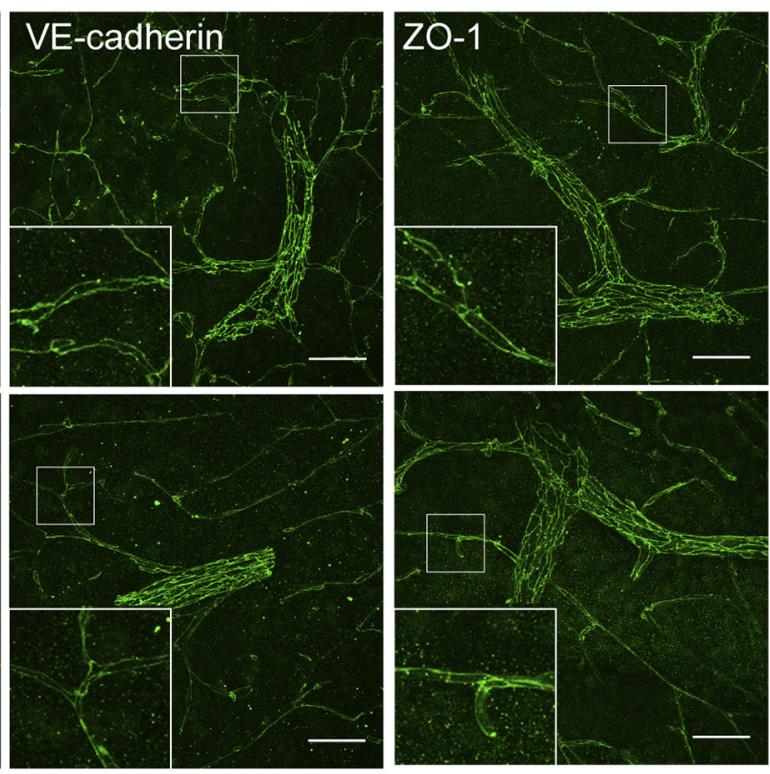

Claudin-5

D

VE-cadherin

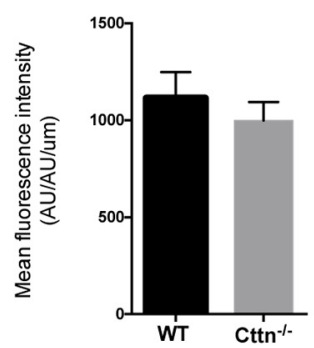

E

$\mathrm{ZO}-1$
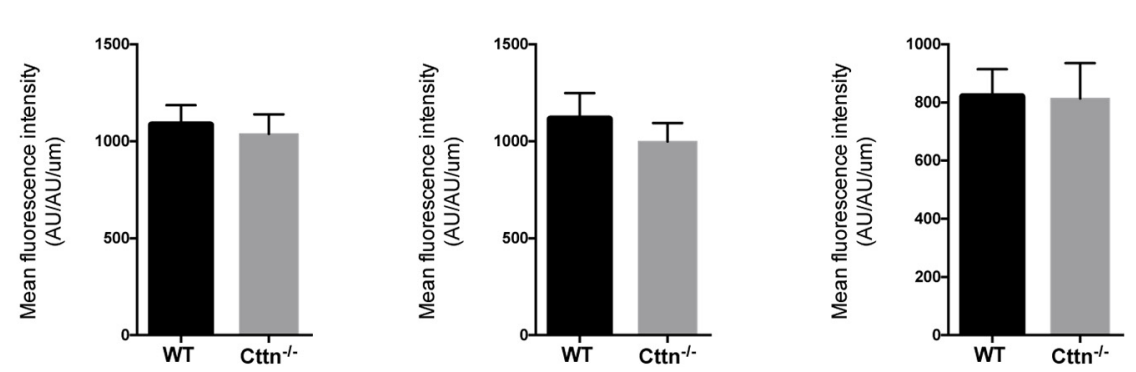

Figure 8. Protein expression of adhesion and tight junction proteins in the brain vasculature of $\mathrm{Cttn}^{-1-}$ and WT mice. $A$, Immunofluorescence staining of MBMECs, isolated from the brains of 5 WT and $\mathrm{Cttn}^{-1-}$ mice, for occludin, VE-cadherin, and ZO-1. B, Whole-mount staining of blood vessels in vibratome sections from healthy WT and $\mathrm{Cttn}^{-1-}$ brains for claudin-5, VE-cadherin, and Z0-1 (insets, higher magnification). Scale bar, $50 \mu \mathrm{m}$. Images are representative for at least three independent preparations each. Mean fluorescence intensity of the staining for junctional proteins in brain sections was quantified for claudin-5(C), VE-cadherin (D), and ZO- 1 (E). Graphs represent mean \pm SEM with $n=8$ WT and $n=10 \mathrm{Cttn}^{-1-}$ mice $(\boldsymbol{C}) ; n=6 \mathrm{WT}$ and $n=8 \mathrm{Cttn}{ }^{-1-}$ mice (D); and $n=8 \mathrm{WT}$ and $n=6 \mathrm{Cttn}^{-1-}$ mice (E). Statistics were analyzed with (C) Mann-Whitney U test, $p=0.3599$; unpaired two-tailed Student's t test: (D) $p=0.4893,(\boldsymbol{E}) p=0.9694$. 
A

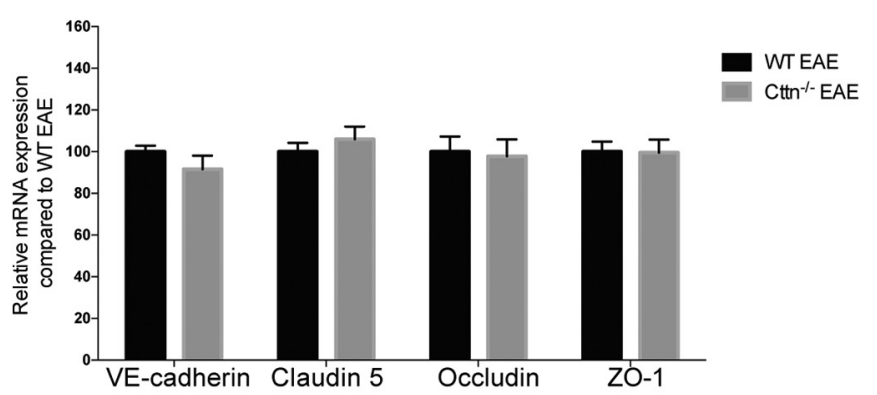

C Adhesion molecules in healthy brain

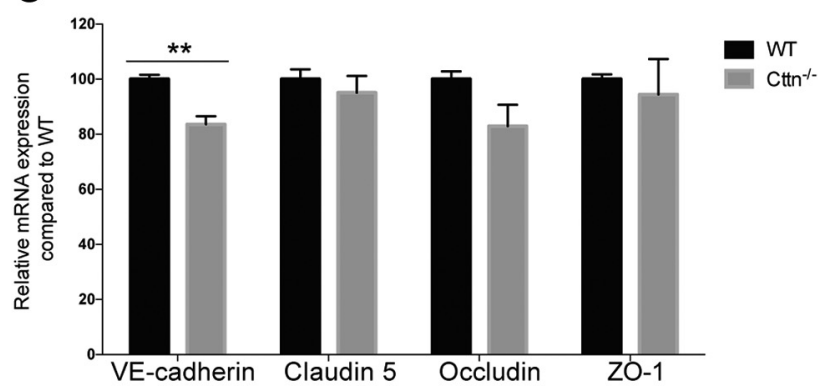

B

Adhesion molecules in EAE spinal cord

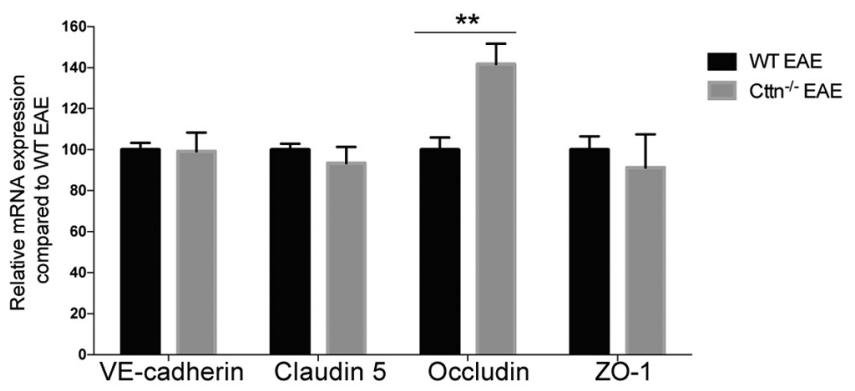

D Adhesion molecules in healthy spinal cord

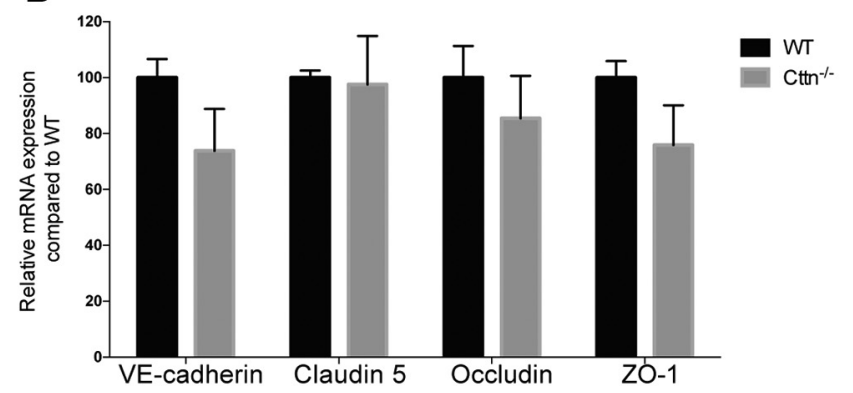

Figure 9. RNA expression of junctional molecules in healthy and inflamed CNS of $\mathrm{Cttn}^{-1-}$ and WT mice. Total mRNA was isolated from $\mathrm{Cttn}^{-1-}$ and WT mice at the peak of EAE $(\boldsymbol{A}, \boldsymbol{B})$ and without EAE $(C, D)$, and gene expression was measured by means of qRT-PCR (normalized to GAPDH expression) with expression levels in WT mice set as $100 \%$. Graphs represent mean \pm SEM of

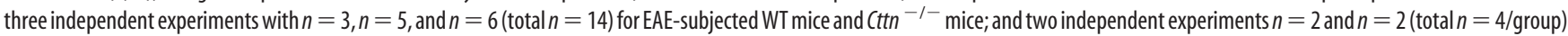
for healthy WT and $\mathrm{Cttn}^{-1-}$ mice. Statistics were analyzed with unpaired two-tailed Student's $t$ test: ${ }^{* *} p=0.002$ (occludin); ${ }^{* *} p=0.005$ (VE-cadherin).

inactivation protects from an increase in vascular permeability in EAE, most likely due to reduced entry of inflammatory cells.

\section{Cortactin supports diapedesis of T cells through mouse primary brain endothelial cells}

Due to the special barrier function of the BBB endothelium, we analyzed the migration of $\mathrm{CD} 4{ }^{+} \mathrm{T}$ cells across monolayers of primary MBMECs in in vitro transmigration assays. First, we confirmed expression of cortactin in WT MBMECs, which was absent in the MBMECs from $\mathrm{Cttn}^{-/-}$mice (Fig. 7). Cortactin was found close to junctions but also throughout the cell. It has been previously reported that silencing of cortactin by shRNA in human brain microvascular endothelial cells leads to fragmented staining of claudin-5, occluding, and ZO-1 (Stamatovic et al., 2015). However, comparison of occludin, VE-cadherin, and $\mathrm{ZO}-1$ staining patterns in of WT and $\mathrm{Cttn}^{-1-}$ primary MBMECs revealed no differences. Likewise, whole-mount staining of blood vessels in vibratome sections of mouse brains from WT and $\mathrm{Cttn}^{-1-}$ mice showed no differences in the junctional expression patterns of claudin-5, VE-cadherin, and ZO-1 (Fig. 8). Analyzing gene expression of claudin-5, VE-cadherin, and ZO-1 by qRTPCR in brain and spinal cord of mice with EAE at the peak of disease and of WT mice revealed no gross changes between the two genotypes either, with the exception of a slight reduction of VE-cadherin levels in the brain of healthy (but not diseased) $\mathrm{Cttn}^{-1-}$ mice and a slight increase of occludin expression in the spinal cord of diseased (but not healthy) $\mathrm{Cttn}^{-1-}$ mice (Fig. 9).

We next tested whether the absence of cortactin affects the diapedesis of $\mathrm{T}$ cells through primary MBMEC monolayers. We found that transmigration through $\mathrm{Cttn}^{-1-}$ brain endothelial cells was indeed reduced compared with WT endothelial cells (Fig. 10A). Interestingly, adhesion of T cells to MBMEC under static conditions was similar for both genotypes (Fig. 10B). However, under flow conditions $\mathrm{T}$ cell adhesion to MBMEC was reduced in the absence of cortactin (Fig. 10C). We conclude that cortactin in mouse brain endothelial cells supports the docking and transmigration of $\mathrm{CD} 4{ }^{+} \mathrm{T}$ cells. Quantifying the expression level of ICAM-1 on primary MBMECs from $\mathrm{Cttn}^{-1-}$ and WT mice showed very weak expression in the absence of cytokines, which was strongly upregulated by TNF- $\alpha$ to similar levels for both genotypes (Fig. 11). Thus, the expression level of ICAM-1 is not affected by cortactin.

\section{Discussion}

Leukocyte extravasation through the BBB is a crucial step in the development of autoimmune demyelinating conditions of the CNS, such as multiple sclerosis and the respective animal model EAE. Yet, in contrast to the endothelium of other organs, the endothelium of the $\mathrm{BBB}$ represents a special barrier that is less easily overcome by solutes and leukocytes. Mechanistic details about the transmigration of leukocytes through the $\mathrm{BBB}$ are less well understood than for the endothelium in other tissues. Here, we show that the actin-binding protein cortactin is required for immune cell infiltration into the CNS in the context of EAE. EAE severity was clearly reduced in cortactin gene inactivated mice. Accordingly, proinflammatory cytokine levels in the CNS were reduced and inflammation-induced vascular permeability was attenuated. Since we could rule out a negative effect of the lack of cortactin on the induction of the immune response, we conclude that interference with endothelial cortactin inhibits the entry of inflammatory cells into the CNS, which was further substantiated by less efficient migration of $\mathrm{CD}^{+} \mathrm{T}$ cells through primary brain endothelial cells isolated from $\mathrm{Cttn}^{-1-}$ mice. 
A

$1 \mathrm{~h}$ after applying $\mathrm{T}$ cells

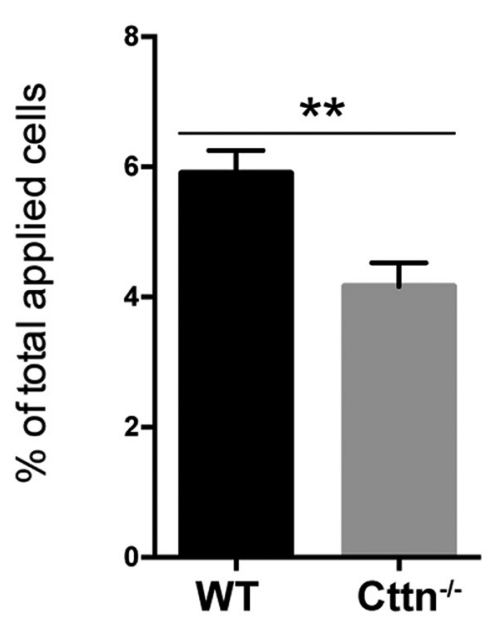

B

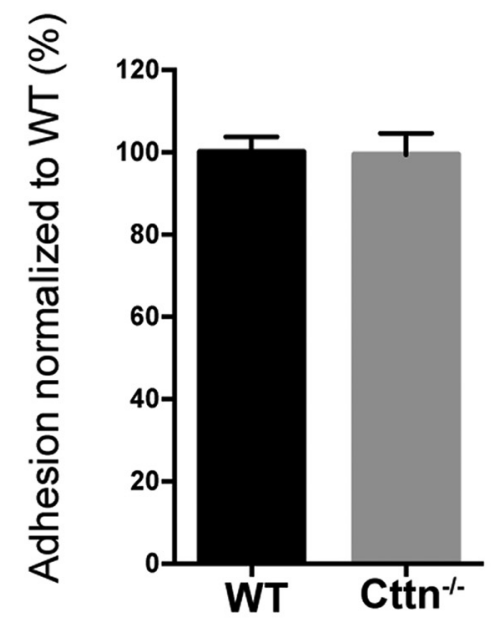

$3.5 \mathrm{~h}$ after applying $\mathrm{T}$ cells

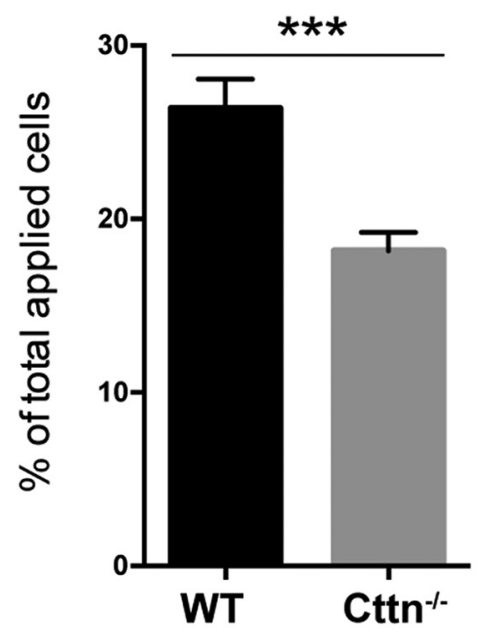

C

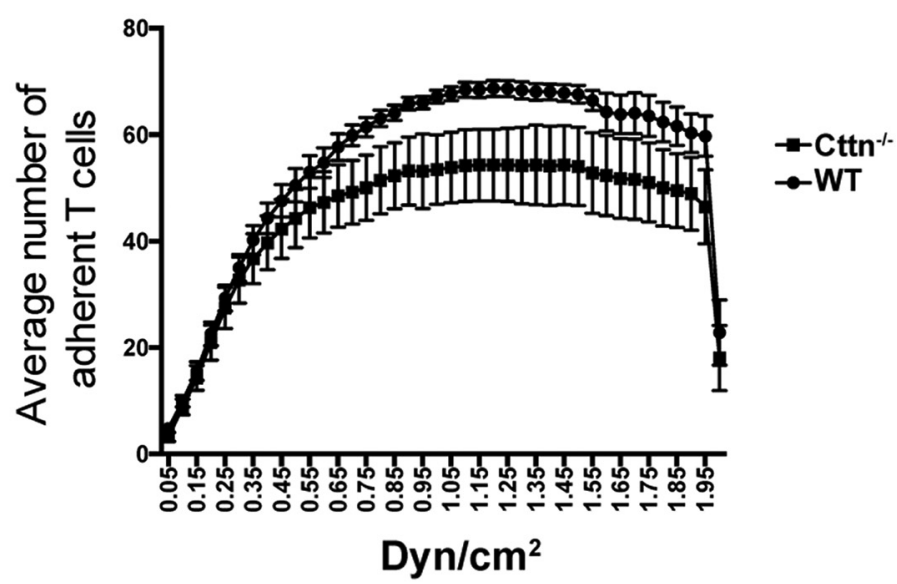

Figure 10. Lack of cortactin impairs T-cell transmigration through primary brain endothelial cells. Assays were performed with MBMECs isolated from WT and $\mathrm{Cttn}^{-1-}$ mice. $\boldsymbol{A}$, Transmigration

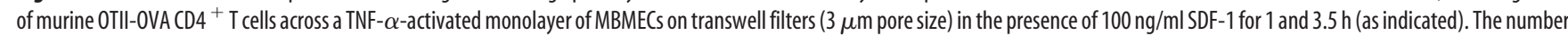
of transmigrated cells is displayed as percentage of total applied cells. Graphs represent mean \pm SEM of three independent experiments with $n=6, n=6$, and $n=7$ (total $n=19$ ) filters for WT; $n=6, n=6$, and $n=8$ (total of $n=20$ ) filters for Cttn ${ }^{-1-}$ cells. B, T cells (as above) were allowed to bind to EC (as above) in a 96-well plate for 30 min. The number of adherent cells to WT EC was set as 100\%. Graph represents mean \pm SEM of two independent experiments with $n=8, n=10$ (total $n=18$ ) for WT and (ttn ${ }^{-1-}$ cells. C, T cells (as above) were passed over EC (as above) under flow conditions increasing from 0 to 2 dyne $/ \mathrm{cm}^{2}$ for 30 min in a 6-channel $\mu$-Slide VI 0.4 (ibidi) in the presence of $50 \mathrm{ng} / \mathrm{ml} \mathrm{SDF-1.} \mathrm{Graph} \mathrm{represents} \mathrm{mean} \pm$ SEM of 20 channels for WT and $\mathrm{Cttn}^{-l-}$ EC. Statistics were analyzed with unpaired two-tailed Student's $t$ test: $\boldsymbol{A}^{* *}{ }^{* *} p=0.0015,{ }^{* * *} p=0.0003 ; \boldsymbol{B}, p=0.9293 ; \boldsymbol{C}, p=0.2324$.

The reduced leukocyte transmigration efficiency across the $\mathrm{BBB}$ of $\mathrm{Cttn}^{-1-}$ mice is reminiscent of our findings with $\mathrm{Cttn}^{-/-}$ primary lung endothelial cells (Schnoor et al., 2011) and shows that, despite the special character of brain endothelial cells, the contribution of cortactin to the transmigration process is of basic importance and relevant in both endothelial cell subtypes. In our previous study, we found that cortactin was required for the clustering of endothelial ICAM-1 around adhering/transmigrating neutrophils at the diapedesis pore, which was dependent on ICAM-1-triggered RhoG activation (Schnoor et al., 2011). Cortactin acted upstream of the activation of RhoG in this process. This is in accordance with other studies demonstrating tyrosine phosphorylation of cortactin upon crosslinking of ICAM-1 (Durieu-Trautmann et al., 1994) and resulting coprecipitation of cortactin with ICAM-1 (Tilghman and Hoover, 2002). In line with this, silencing of cortactin in cultured endothelial cells inhibits accumulation of ICAM- 1 at sites of neutrophil attachment and inhibits transmigration (Yang et al., 2006a,b). The latter study suggested that silencing of cortactin does not impair adhesion of neutrophils to EC in assays under static conditions. However, in vivo, we have shown that neutrophil adhesion and extravasation are reduced in postcapillary venules of the cremaster in the absence of cortactin, arguing that cortactin-dependent ICAM-1 clustering might be needed for neutrophil arrest as well as for the transmigration process under physiological flow conditions (Schnoor et al., 2011). Since ICAM-1 is also an important player for the entry of leukocytes into the CNS (Lyck and Engelhardt, 2012), it may be that cortactin-dependent clustering of ICAM-1 is also needed in postcapillary venules of the brain for leukocyte extravasation. 
In vivo evidence for the relevance of cortactin for leukocyte extravasation was so far limited to neutrophils. Our flow cytometry analyses in the present study revealed a decrease in all measured leukocyte types that had infiltrated into the brain of $\mathrm{Cttn}^{-1-}$ mice, including $\mathrm{CD} 4{ }^{+}$ and $\mathrm{CD} 8{ }^{+} \mathrm{T}$ cells, macrophages, and neutrophils. We conclude therefore that cortactin contributes to basic mechanisms of the transmigration process, which are common to a large repertoire of leukocyte types.

Of interest, we detected in $\mathrm{Cttn}^{-1-}$ mice a selective inhibition of Th17 lymphocyte recruitment to spinal cord, whereas Th1 cell recruitment was as in WT mice. Considering that caveolar transcytosis was reported to be required for Th1, but not Th17, cell entry into the CNS (Lutz et al., 2017), our results may suggest that cortactin is needed for a junction-related extravasation route, which may be more relevant for Th17 cells. This may warrant future studies to elucidate a possible role of cortactin in the paracellular diapedesis of leukocytes.

We have previously shown that cortactin contributes to vascular integrity because cortactin deficiency led to increased basal vascular permeability in the skin and the permeability-enhancing effect of histamine was substantially increased in cortactin gene inactivated mice compared with WT mice (Schnoor et al., 2011). In agreement with these results, we show here that the absence of cortactin caused increased basal vascular permeability in the spinal cord blood vessels. By contrast, we found no such effect in the brain or in the kidney, highlighting the fact that regulatory elements determining baseline junctional integrity varies between vascular beds of different organs. Despite the junction stabilizing function of cortactin, the lack of cortactin protected from vascular leak formation in brain and spinal cord during EAE. This is probably because cerebral permeability tightly follows leukocyte extravasation (Leibowitz and Kennedy, 1972; Butter et al., 1991). Transmigration of immune cells through the $\mathrm{BBB}$ contributes to an increase in vascular permeability in a number of ways: by upregulation of the production and secretion of proinflammatory cytokines, changes in factors, such as matrix metalloproteinase activity, reactive oxygen species, histamine, and neurotrophins. These factors contribute to the disruption of the molecular organization of the BBB and increase in the expression of adhesion molecules and chemoattractants by the brain
A

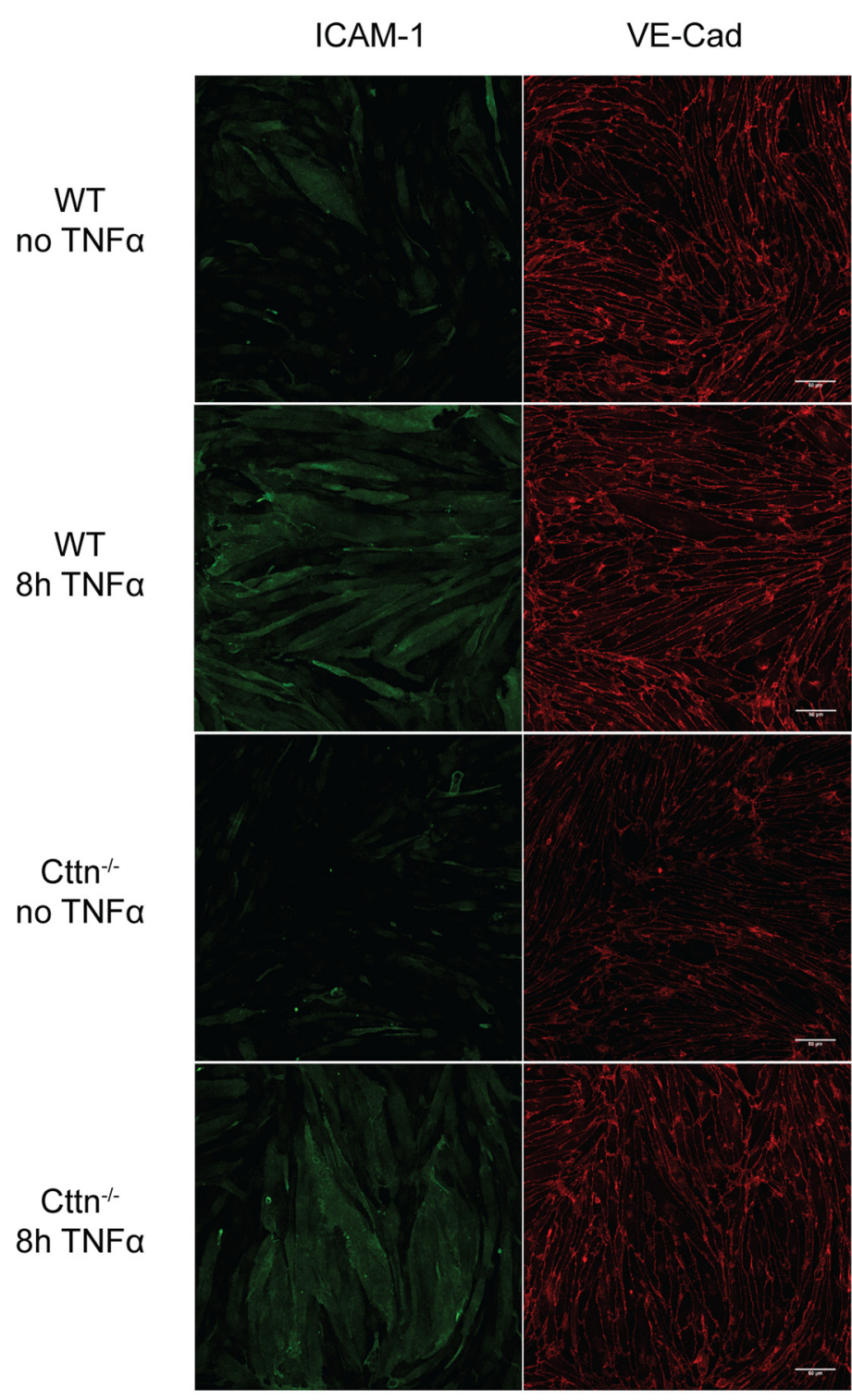

B

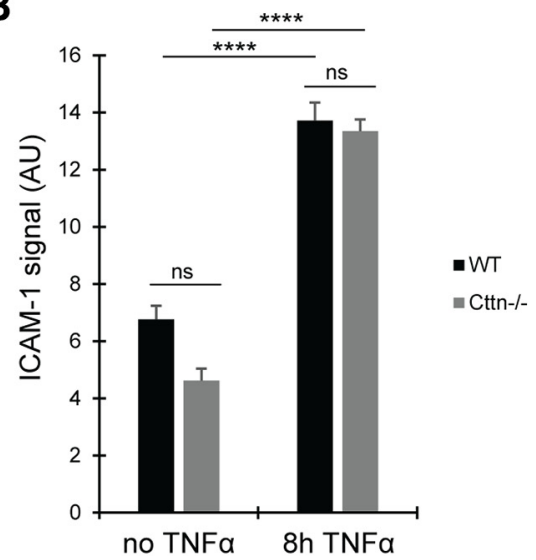

Figure 11. ICAM-1 expression in primary brain endothelial cells after TNF $\alpha$ is not affected by cortactin deficiency. $A, A$ representative immunofluorescence staining of MBMEC of indicated genotypes stimulated or unstimulated for $8 \mathrm{~h}$ with TNF $\alpha$. Staining was performed with antibodies against ICAM-1 and VE-cadherin (VE-Cad). Scale bar, $50 \mu \mathrm{m}$. B, Quantification of ICAM-1 expression per unit area was performed with ImageJ. Data are mean \pm SEM of 16 (unstimulated) or 32 (stimulated) measurement areas $\left(0.01 \mathrm{~mm}^{2}\right)$ per genotype. ${ }^{* * *} p<0.001, \mathrm{~ns}=$ not significant, Kruskal-Wallis one-way ANOVA on Ranks was performed $(\mathrm{H}=$ $60.061, \mathrm{df}=3$ ) followed by Dunn's test. 
microvascular endothelial cells, thereby perpetuating the infiltration of immune cells into the CNS (Larochelle et al., 2011). In accordance with this hypothesis, we found reduced levels of proinflammatory cytokines in brains and spinal cords of $\mathrm{Cttn}^{-1-}$ mice. Therefore, we suggest that the decrease in vascular permeability observed in $\mathrm{Cttn}^{-1-}$ mice during EAE is due to the reduced infiltration of immune cells into the CNS and the resulting lower levels of BBB disrupting proinflammatory mediators. This positive correlation between the transmigration of leukocytes and the induction of permeability on the tissue level should not be mistaken with a direct effect of the diapedesis process on junctional leakiness. It is now well documented that the diapedesis process does not per se cause enhanced leakiness (Cutler et al., 1967; Leibowitz and Kennedy, 1972; Baluk et al., 1998; Schnoor et al., 2011; Wessel et al., 2014; Heemskerk et al., 2016). The supportive effect of cortactin inactivation on permeability induction in EAE is most likely based on the reduced levels of proinflammatory cytokines, which is caused by the reduced numbers of inflammatory leukocytes in the CNS.

An earlier study reported on the role of cortactin in the arrangement of molecular components of endothelial junctions. Silencing of cortactin in human brain microvascular endothelial cell cultures by shRNA caused fragmented staining of claudin-5, occluding, and ZO-1 (Stamatovic et al., 2015). Contrary to this study, we did not observe differences in the stainings for occludin, ZO-1, or VE-cadherin between primary brain endothelial cells isolated from $\mathrm{Cttn}^{-1-}$ and WT mice. Also, whole-mount stainings of vibratome sections of the brain of $\mathrm{Cttn}^{-1-}$ and WT mice for claudin-5, VE-cadherin, and ZO-1 showed no differences. Whether this is due to species differences or other reasons is presently unknown.

In conclusion, we have shown that interference with the expression of cortactin protects from the induction of clinical symptoms of EAE by attenuating the entry of inflammatory leukocytes into the CNS of EAE mice. These results establish cortactin as a potential target for reagents interfering with EAE, a model for multiple sclerosis.

\section{References}

Alon R, van Buul JD (2017) Leukocyte breaching of endothelial barriers: the actin link. Trends Immunol 38:606-615.

Baluk P, Bolton P, Hirata A, Thurston G, McDonald DM (1998) Endothelial gaps and adherent leukocytes in allergen-induced early- and late-phase plasma leakage in rat airways. Am J Pathol 152:14631476.

Broermann A, Winderlich M, Block H, Frye M, Rossaint J, Zarbock A, Cagna G, Linnepe R, Schulte D, Nottebaum AF, Vestweber D (2011) Dissociation of VE-PTP from VE-cadherin is required for leukocyte extravasation and for VEGF-induced vascular permeability in vivo. J Exp Med 208: 2393-2401.

Butter C, Baker D, O’Neill JK, Turk JL (1991) Mononuclear cell trafficking and plasma protein extravasation into the CNS during chronic relapsing experimental allergic encephalomyelitis in Biozzi AB/H mice. J Neurol Sci 104:9-12.

Cosen-Binker LI, Kapus A (2006) Cortactin: the gray eminence of the cytoskeleton. Physiology (Bethesda) 21:352-361.

Cutler RW, Lorenzo AV, Barlow CF (1967) Brain vascular permeability to I-125 gamma globulin and leukocytes in allergic encephalomyelitis. J Neuropathol Exp Neurol 26:558-571.

Dudek SM, Jacobson JR, Chiang ET, Birukov KG, Wang P, Zhan X, Garcia JG (2004) Pulmonary endothelial cell barrier enhancement by sphingosine 1-phosphate: roles for cortactin and myosin light chain kinase. J Biol Chem 279:24692-24700.

Durieu-Trautmann O, Chaverot N, Cazaubon S, Strosberg AD, Couraud PO (1994) Intercellular adhesion molecule 1 activation induces tyrosine phosphorylation of the cytoskeleton-associated protein cortactin in brain microvessel endothelial cells. J Biol Chem 269:1253612540 .

Fenstermacher J, Gross P, Sposito N, Acuff V, Pettersen S, Gruber K (1988) Structural and functional variations in capillary systems within the brain. Ann N Y Acad Sci 529:21-30.

García Ponce A, Citalán Madrid AF,Vargas Robles H, Chánez Paredes S, Nava P, Betanzos A, Zarbock A, Rottner K, Vestweber D, Schnoor M (2016) Loss of cortactin causes endothelial barrier dysfunction via disturbed adrenomedullin secretion and actomyosin contractility. Sci Rep 6:29003.

Heemskerk N, Schimmel L, Oort C, van Rijssel J, Yin T, Ma B, van Unen J, Pitter B, Huveneers S, Goedhart J, Wu Y, Montanez E, Woodfin A, van Buul JD (2016) F-actin-rich contractile endothelial pores prevent vascular leakage during leukocyte diapedesis through local RhoA signalling. Nat Commun 7:10493.

Jacobson JR, Dudek SM, Singleton PA, Kolosova IA, Verin AD, Garcia JG (2006) Endothelial cell barrier enhancement by ATP is mediated by the small GTPase Rac and cortactin. Am J Physiol Lung Cell Mol Physiol 291:L289-L295.

Larochelle C, Alvarez JI, Prat A (2011) How do immune cells overcome the blood-brain barrier in multiple sclerosis? FEBS Lett 585:37703780.

Leibowitz S, Kennedy L (1972) Cerebral vascular permeability and cellular infiltration in experimental allergic encephalomyelitis. Immunology 22: 859-869.

Li-ChunHsieh K, Schob S,Zeller MW, Pulli B, Ali M, Wang C, Chiou TT, Tsang YM, Lee PS, Stossel TP, Chen JW (2015) Gelsolin decreases actin toxicity and inflammation in murine multiple sclerosis. J Neuroimmunol 287:36-42.

Lutz SE, Smith JR, Kim DH, Olson CV, Ellefsen K, Bates JM, Gandhi SP, Agalliu D (2017) Caveolin1 is required for Th1 cell infiltration, but not tight junction remodeling, at the blood-brain barrier in autoimmune neuroinflammation. Cell Rep 21:2104-2117.

Lyck R, Engelhardt B (2012) Going against the tide: how encephalitogenic T cells breach the blood-brain barrier. J Vasc Res 49:497-509.

Mullins RD, Heuser JA,Pollard TD (1998) The interaction of Arp2/3 complex with actin: nucleation, high affinity pointed end capping, and formation of branching networks of filaments. Proc Natl Acad Sci U S A 95: 6181-6186.

Nourshargh S, Alon R (2014) Leukocyte migration into inflamed tissues. Immunity 41:694-707.

Ransohoff RM, Engelhardt B (2012) The anatomical and cellular basis of immune surveillance in the central nervous system. Nat Rev Immunol 12:623-635.

Ruck T, Bittner S, Epping L, Herrmann AM, Meuth SG (2014) Isolation of primary murine brain microvascular endothelial cells. J Vis Exp 93:6-11.

Schnoor M, Lai FP, Zarbock A, Kläver R, Polaschegg C, Schulte D, Weich HA, Oelkers JM, Rottner K, Vestweber D (2011) Cortactin deficiency is associated with reduced neutrophil recruitment but increased vascular permeability in vivo. J Exp Med 208:1721-1735.

Schnoor M, Stradal TE, Rottner K (2018) Cortactin: cell functions of A multifaceted actin-binding protein. Trends Cell Biol 28:79-98.

Sedlakova R, Shivers RR, Del Maestro RF (1999) Ultrastructure of the bloodbrain barrier in the rabbit. J Submicrosc Cytol Pathol 31:149-161.

Sixt M, Engelhardt B, Pausch F, Hallmann R, Wendler O, Sorokin LM (2001) Endothelial cell laminin isoforms, laminins 8 and 10, play decisive roles in $\mathrm{T}$ cell recruitment across the blood-brain barrier in experimental autoimmune encephalomyelitis. J Cell Biol 153:933-946.

Sorokin L (2010) The impact of the extracellular matrix on inflammation. Nat Rev Immunol 10:712-723.

Stamatovic SM, Sladojevic N,Keep RF, Andjelkovic AV (2015) PDCD10 (CCM3) regulates brain endothelial barrier integrity in cerebral cavernous malformation type 3: role of CCM3-ERK1/2-cortactin cross-talk. Acta Neuropathol 130:731-750. 
Tilghman RW, Hoover RL (2002) The src-cortactin pathway is required for clustering of E-selectin and ICAM-1 in endothelial cells. FASEB J 16:1257-1259.

Uruno T, Liu J, Zhang P, Fan Y, Egile C, Li R, Mueller SC, Zhan X (2001) Activation of Arp2/3 complex-mediated actin polymerization by cortactin. Nat Cell Biol 3:259-266.

van Rossum AG, van Buuren-van Seggelen V, Kluin PM, Schuuring E (2005) Comparative genome analysis of cortactin and HS1: the significance of the F-actin binding repeat domain. BMC Genomics 6:15.

Vestweber D (2015) How leukocytes cross the vascular endothelium. Nat Rev Immunol 15:692-704.

Wessel F, Winderlich M, Holm M, Frye M, Rivera-Galdos R, Vockel M,
Linnepe R, Ipe U, Stadtmann A, Zarbock A, Nottebaum AF, Vestweber D (2014) Leukocyte extravasation and vascular permeability are each controlled in vivo by a different tyrosine residue of VE-cadherin. Nat Immunol 15:223-230.

Yang L, Kowalski JR, Zhan X, Thomas SM, Luscinskas FW (2006a) Endothelial cell cortactin phosphorylation by Src contributes to polymorphonuclear leukocyte transmigration in vitro. Circ Res 98:394-402.

Yang L, Kowalski JR, Yacono P, Bajmoczi M, Shaw SK, Froio RM, Golan DE, Thomas SM, Luscinskas FW (2006b) Endothelial cell cortactin coordinates intercellular adhesion molecule-1 clustering and actin cytoskeleton remodeling during polymorphonuclear leukocyte adhesion and transmigration. J Immunol 177:6440-6449. 